\title{
Optimal Q-laws via Reinforcement Learning with Guaranteed Stability
}

\author{
Harry Holt ${ }^{\mathrm{a}}$, Roberto Armellin ${ }^{\mathrm{b}}$, Nicola Baresi ${ }^{\mathrm{c}}$, Yoshi Hashida ${ }^{\mathrm{d}}$, Andrea \\ Turconi $^{\mathrm{d}}$, Andrea Scorsoglio ${ }^{\mathrm{e}}$, Roberto Furfaro ${ }^{\mathrm{f}}$ \\ ${ }^{a}$ PhD Student, Surrey Space Centre, University of Surrey, GU2 7XH, Guildford, United \\ Kingdom \\ ${ }^{b}$ Professor, Te Pūnaha Ātea - Auckland Space Institute, University of Auckland, \\ Auckland, New Zealand \\ ${ }^{c}$ Lecturer, Surrey Space Centre, University of Surrey, GU2 7XH, Guildford, United \\ Kingdom \\ ${ }^{d}$ AOCS Engineer, Surrey Satellite Technology Limited, 20 Stephenson Rd, Guildford \\ GU2 7YE, United Kingdom \\ ${ }^{e}$ PhD Student, Department of Systems and Industrial Engineering, University of \\ Arizona, Tuscon, AZ 85721, USA \\ ${ }^{f}$ Professor, Department of Systems and Industrial Engineering, University of Arizona, \\ Tuscon, AZ 85721, USA
}

\begin{abstract}
Closed-loop feedback-driven control laws can be used to solve low-thrust many-revolution trajectory design and guidance problems with minimal computational cost. Lyapunov-based control laws offer the benefits of increased stability whilst their optimality can be increased by tuning their parameters. In this paper, a reinforcement learning framework is used to make the parameters of the Lyapunov-based Q-law state-dependent, increasing its optimality. The Jacobian of these state-dependent parameters is available analytically and, unlike in other optimisation approaches, can be used to enforce stability throughout the transfer. The results focus on GTO-GEO and LEO-GEO transfers in Keplerian dynamics, including the effects of eclipses. The impact of the network architecture on the behaviour is investigated for both time- and mass-optimal transfers. Robustness to navigation errors and thruster misalignment is demonstrated using Monte Carlo analyses. The resulting approach offers potential for on-board autonomous transfers and
\end{abstract}

\footnotetext{
*Corresponding author: h.holt@surrey.ac.uk
} 
orbit reconfiguration.

Keywords: Low-thrust, Reinforcement Learning, Lyapunov Control, State-dependent, Stability

\section{Introduction}

Low-thrust many-revolution trajectory design is becoming increasingly important with the development of high specific impulse, low-thrust engines such as electric propulsion (EP) systems, with 243 satellites launching with EP systems as of 2017. ${ }^{1}$ Initially used for station-keeping tasks, EP is now increasingly used for orbit raising applications, demonstrated in full by the SMART-1 satellite in 2003, when it flew to the Moon, and confirmed by the successful flight of Eutelsat 172B in June 2017, taking approximately four months to rise from GTO to GEO. ${ }^{2}$ The technology, along with solar power sails (JAXA's IKAROS and OKEANOS missions ${ }^{3}$ ) is being increasingly used in both planetary and interplanetary spacecraft (NASA's Dawn mission and JAXA's Hayabusa 1 \& 2).

The near continuous thrust has resulted in the need for trajectory design techniques that can handle both low control authority and long burn arcs. This increase in computational difficultly impacts both mission design phases and future on-board automatic guidance. Initial work on solving low-thrust trajectories began with Edelbaum's work in the $1960 \mathrm{~s}^{4}$ and now conventional direct and indirect optimisation methods are available and can solve these complex optimal control problems. ${ }^{5,6}$ However, they are computationally intensive and still present many challenges, particularly regarding initial guesses and convergence. Both methods provide point solutions (i.e., for the assumed initial condition) and cannot be used as guidance laws due to time limitations and difficulties in ensuring convergence, especially on limited on-board computers. ${ }^{7}$

Closed-loop feedback-driven (CLFD) control laws allow the computation of sub-optimal trajectories with minimal computational cost. ${ }^{8,9,10}$ They consider the trajectory design problem from a targeting perspective rather than an optimisation one, and only require knowledge of the current spacecraft state and target state in order to determine the control to be applied. This makes them suitable as initial guesses for indirect and direct methods ${ }^{11,12}$ and they have great potential for on-board use. 
Given the importance stability plays in spacecraft trajectory design, many CLFD control laws are based on Lyapunov control theory ${ }^{13}$ as opposed to thrust-blending ones such as the Directional Adaptive Guidance algorithm. ${ }^{14}$ Joseph, ${ }^{15}$ Naasz $^{16}$ and Petropoulos' Q-law ${ }^{9,10}$ are all examples of Lyapunov controllers. Several works have further extended the capabilities of these control laws in the presence of perturbations. In 2008, Maddock and Vasile ${ }^{17}$ extended a Lyapunov-based control law to handle both solar radiation pressure and $3^{\text {rd }}$-body effects. Baresi et al. $2018^{18}$ derived a Lyapunov controller using relative orbital elements for orbit maintenance around the Martian moon Phobos in a similar fashion to Ref. 13. Epenoy and Pérez-Palau ${ }^{19}$ combined one with an invariant manifold approach for Earth-Moon transfers. This also included solar radiation pressure and third-body effects. Pontani et al. $2021^{20}$ have explored their stability in the presence of perturbations for the station-keeping and guidance problem.

However, Lyapunov controllers are inherently sub-optimal and have many user-defined parameters which significantly affect their performance. ${ }^{21,22,23}$ One method of achieving this is using evolutionary algorithms (EAs). The multi-objective nature of spacecraft trajectory design lends itself to EAs and they still provide many of the benchmarks in the area. ${ }^{24}$ Both Lee et al. $2005^{21}$ and Varga et al. 2016 ${ }^{22}$ used a multi-objective genetic algorithm to optimise the Q-law design parameters for a variety of Earth orbit transfers, with the design parameters remaining fixed throughout the transfer. Yang et al. ${ }^{25}$ used an artificial neural network and improved cooperative evolutionary algorithm optimiser to make the design parameters of a Q-law state-dependent.

We aim to increase the optimality and retain the stability of CLFD control laws using reinforcement learning (RL) methods. The goal is to develop a lightweight and closed-loop control law that can be used for both initial trajectory design and subsequent on-board guidance. The major draw for RL algorithms is their performance in unfamiliar environments. Thus, there is significant interest in the application of RL in astrodynamics, from mission design, operations, guidance and control to navigation and even the prediction of the dynamics. ${ }^{24}$ Gaudet, Furfaro and Linares have demonstrated its capability for use in Mars and Lunar landers ${ }^{26,27}$ and asteroid hovering missions. ${ }^{28,29}$ Several works are using RL as a tool for trajectory design and guidance in the circular restricted three-body problem. ${ }^{30,31,32,33}$ There has been success for similar approaches in rendezvous and landing problems. $^{34,35,36}$ Kwon et al. $2021^{37}$ have recently used RL as a tool for 
many-revolution transfers. However, little work has combined RL with these CLFD control laws for trajectory design specifically, which would result in a powerful state-dependent and more optimal controller.

The work in this paper presents a stable Lyapunov controller combined with a reinforcement learning architecture. The advantage of combining these two approaches allows one to eliminate the drawbacks from each approach: namely the sub-optimality of Lyapunov controllers and the unknown stability of RL methods. The authors demonstrated a prototype RL architecture and control approach in conference proceedings, ${ }^{38,39}$ showing potential for space-borne applications. Here we present a state-dependent controller which enforces stability without compromising optimality through the Jacobian of the state-dependent parameters. Performance for both time and mass-optimal transfers are discussed, and the influence of the RL architecture on both optimality and stability is presented for the first time. To the author's knowledge the addition of stability through the Jacobian term has not been explored elsewhere, the importance of which can extend beyond trajectory design and more generally to optimisation of non-linear controllers.

Although out-of-scope for this work, this approach also offers substantial potential for on-board use. Currently a classical Lyapunov controller could be implemented on-board as a guidance closed-loop control law. What we propose is to train a neural network on-ground and upload the trained network alongside the Lyapunov controller. This retains a closed-loop controller with much improved optimality, saving on either time-of-flight or propellant mass. The computationally expensive learning process is thus separated from the resulting on-board controller thanks to the trained neural network. Once trained, the closed-loop nature of the neural networks offers the ability to quickly and autonomously evaluate a control history for the spacecraft with basic linear algebra operations.

The paper is structured as follows. First the Q-law control theory is discussed, showing the two types of control considered. The Jacobian derivation is given in the following section, and comprises the major novel contribution of this work. Key concepts in RL are discussed, along with the particular implementation used. The results for transfers from geostationary transfer orbit to geostationary orbit (GTO-GEO) and low-Earth orbit to geostationary orbit (LEO-GEO) are presented for both time and mass-optimal transfers in Keplerian dynamics with and without eclipse effects. These transfers are selected to offer comparison with previous work in the literature. A particle swarm optimisation (PSO) is used to generate time-dependent Q-laws 
which act as a suitable benchmark for comparison with the state-dependent RL approach. Robustness to navigation errors and thruster misalignment is demonstrated using Monte Carlo analyses.

\section{Theory}

The proposed approach can be broken down into three sections: the dynamics and control, the state-dependence, and the RL algorithm. The controller is based on the work of Petropoulos and hereby derived from a "Q-law" Lyapunov function. ${ }^{9,10}$ In order to improve the optimality of the controller without loss of stability, the control parameters are made state-dependent using a neural network (the actor network) to retain the closed-loop nature. Finally, in order to learn this state-dependent nature, a proximal policy optimisation (PPO) algorithm is used.

\subsection{Dynamics}

The spacecraft's motion about a central body is described in terms of the classical orbit element (COEs) semi-major axis $a$, eccentricity $e$, inclination $i$, right ascension of the ascending node (RAAN) $\Omega$ and argument of periapsis $\omega$. If the perturbing acceleration $\boldsymbol{a}_{d}$ is described in the radial, transverse and normal (RTN) frame, then the set of variational equations in $a, e, i, \Omega, \omega$ and the true anomaly $\nu$ take Gauss's form of the Lagrange Planetary Equations. ${ }^{40}$ These can be expressed as:

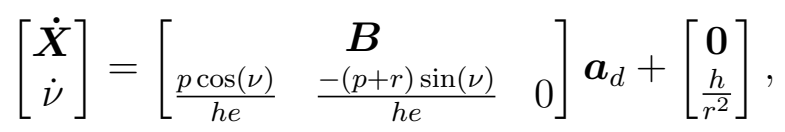

where $\dot{\boldsymbol{X}}$ represents the dynamics of the COE state, $p=a\left(1-e^{2}\right)$ is the semi-latus rectum, $\mu$ the gravitational parameter and $h=\sqrt{\mu p}$. The matrix $\boldsymbol{B}$ represents the Gauss Variational Equations (GVEs) for the slow variables and is required later to compute the control.

$$
\boldsymbol{B}=\left[\begin{array}{ccc}
\frac{2 a^{2}}{h} e \sin (\nu) & \frac{2 a^{2}}{h} \frac{p}{r} & 0 \\
\frac{1}{h} p \sin (\nu) & \frac{1}{h}((p+r) \cos (\nu)+r e) & 0 \\
0 & 0 & \frac{r \cos (\omega+\nu)}{h} \\
0 & 0 & \frac{r \sin (\omega+\nu)}{h \sin (i)} \\
-\frac{1}{h e} p \cos (\nu) & \frac{1}{h e}(p+r) \sin (\nu) & -\frac{r \sin (\omega+\nu) \cos (i)}{h \sin (i)}
\end{array}\right]
$$


When examining Eq. (2), it is clear that singularities occur when $i=0$ or $e=0$. The modified equinoctial elements (MEEs) $p, f, g, h, k$ and fast variable $L$ are used in the dynamical integrator instead of COEs to avoid these issues. ${ }^{41}$ However, the control remains in COEs in order to preserve the physical interpretation of the variables and extract insights from the observed behaviour.

\subsection{Control: Q-law}

The Q-law is best thought of as a weighted, squared summation of the time required to change the current state $\boldsymbol{X}=[a, e, i, \Omega, \omega]^{\mathrm{T}}$ to the target state $\boldsymbol{X}_{T}=\left[a_{T}, e_{T}, i_{T}, \Omega_{T}, \omega_{T}\right]^{\mathrm{T}}$. It can be written as

$$
Q=\left(1+W_{P} P(\boldsymbol{X})\right) \sum_{X} S_{X}(\boldsymbol{X}) W_{X}(\boldsymbol{X})\left(\frac{\delta\left(X, X_{T}\right)}{\max _{\nu}(\dot{X})}\right)^{2},
$$

where $W_{P}$ and $P$ form a penalty function and $S_{X}$ are scaling functions. These are functions of the state and can be found in $\operatorname{Ref} 10 . \delta\left(X, X_{T}\right)=$ $X-X_{T}$ for $X=a, e, i$ whilst $\delta\left(X, X_{T}\right)=\arccos \left(\cos \left(X-X_{T}\right)\right)$ for $X=\Omega, \omega$. The expressions $\max _{\nu}(\dot{X})$ are the maximum rate of change of each COE over the current osculating orbit and can be calculated analytically for all elements except $\omega$. For a more detailed breakdown of the components of the Q-law, the reader is encouraged to look at Refs. 10, 38 and 42. The weights $W_{X}$ can be used to prioritise which elements to target, and are made state-dependent using a neural network, maintaining the closed-loop nature. All other user-defined parameters (for instance, those in $W_{P}, P$ and $S_{X}$ ) are assumed constant, as these terms are only activated to prevent particular behaviour that we also want to prevent. The values are given in Table 1. Hence, the Q-law function becomes a scalar function of both the state $\boldsymbol{X}$, target state $\boldsymbol{X}_{T}$ and weights $\boldsymbol{W}(\boldsymbol{X})$. As written above, the Q-law is a proportional function and as such $\boldsymbol{W}$ are normalised.

Lyapunov's second theorem states that for a system $\dot{\boldsymbol{Z}}=\boldsymbol{f}(\boldsymbol{Z}), \boldsymbol{Z}=$ $\boldsymbol{X}-\boldsymbol{X}_{\mathrm{T}}$, the equilibrium point $\boldsymbol{X}_{\mathrm{T}}$ is asymptotically stable if there exists a scalar Lyapunov function $Q(\boldsymbol{Z})$ such that $Q(\mathbf{0})=0$; it is positive-definite $(Q(\boldsymbol{Z})>0, \forall Z \neq \mathbf{0})$; the derivative is negative-definite $(\dot{Q}(\boldsymbol{Z})<0, \forall Z \neq \mathbf{0})$; and $\lim _{|\boldsymbol{Z}| \rightarrow \infty}, Q(\boldsymbol{Z})=\infty$. $^{13} \mathrm{~A}$ very thorough discussion on the implications of this for trajectory design using nonlinear control can be found in Ref. 20.

Therefore, for the Q-law a stable control is one that ensures $\dot{Q}<0$ throughout the transfer. One way of doing this is to select a controller 
that minimises the rate of change of the Lyapunov function (in this case the most negative value). Classically, this derivative is only a function of state $\boldsymbol{X}$, but that is no longer the case as soon as we allow the Q-law weights to vary. More specifically, if we assume that the weights are state dependent, $\boldsymbol{W}(\boldsymbol{X})$, a new term in the total derivative of the Lyapunov function suddenly emerges:

$$
\begin{aligned}
\dot{Q} & =\dot{Q}_{\boldsymbol{X}}+\dot{Q}_{\boldsymbol{W}} \\
& =\frac{\partial Q}{\partial \boldsymbol{X}} \dot{\boldsymbol{X}}+\frac{\partial Q}{\partial \boldsymbol{W}} \dot{\boldsymbol{W}} \\
& =\frac{\partial Q}{\partial \boldsymbol{X}} \dot{\boldsymbol{X}}+\frac{\partial Q}{\partial \boldsymbol{W}} \frac{\partial \boldsymbol{W}}{\partial \boldsymbol{X}} \dot{\boldsymbol{X}} \\
& =\left(\frac{\partial Q}{\partial \boldsymbol{X}}+\frac{\partial Q}{\partial \boldsymbol{W}} \frac{\partial \boldsymbol{W}}{\partial \boldsymbol{X}}\right) \boldsymbol{B u}
\end{aligned}
$$

where a control vector $\boldsymbol{u}$ is acting as the perturbing acceleration $\boldsymbol{a}_{d}$ in Eq. (1).

We highlight that, when the weights are held constant, $\partial \boldsymbol{W} / \partial \boldsymbol{X}=\mathbf{0}$ and the control $\left(\boldsymbol{u}_{\text {original }}\right)$ is derived as in Eq. (5). However, when $\partial \boldsymbol{W} / \partial \boldsymbol{X} \neq \mathbf{0}$ the control can be modified to account for this as in Eq. (6), or the contribution can be neglected. Given an engine thrust $T$ and a spacecraft mass $m$, the two control vectors are computed as (using $f=T / m$ ):

$$
\begin{gathered}
\boldsymbol{u}_{\text {original }}=-f \frac{\boldsymbol{B}^{\mathrm{T}} \boldsymbol{M}^{\mathrm{T}}}{\|\boldsymbol{M} \boldsymbol{B}\|} \quad \text { where } \quad \boldsymbol{M}=\left(\frac{\partial Q}{\partial \boldsymbol{X}}\right) \\
\boldsymbol{u}_{\text {jacobian }}=-f \frac{\boldsymbol{B}^{\mathrm{T}} \boldsymbol{M}^{\mathrm{T}}}{\|\boldsymbol{M} \boldsymbol{B}\|} \quad \text { where } \quad \boldsymbol{M}=\left(\frac{\partial Q}{\partial \boldsymbol{X}}+\frac{\partial Q}{\partial \boldsymbol{W}} \frac{\partial \boldsymbol{W}}{\partial \boldsymbol{X}}\right) .
\end{gathered}
$$

Coasting can be introduced using effectivity thresholds $\eta^{\text {thresh }}$. These attempt to quantify the effectivity of changing an orbital parameter at a given point in an orbit compared to the optimum point for changing the same orbital parameter. Definitions for both the absolute and relative effectivity parameters exist. Studies ${ }^{21}$ have also shown that, when varying other Q-law parameters in addition to the effectivity, there is little difference in performance between relative and absolute effectivity, and instead the specific transfer will determine which is more applicable. Hence, in this work the absolute effectivity:

$$
\eta_{a}=\frac{\min _{\alpha, \beta}(\dot{Q})}{\min _{\nu}\left(\min _{\alpha, \beta}(\dot{Q})\right)}
$$


is used. $\alpha$ and $\beta$ are the in-plane and out-of-plane angles of the thrust vector, whilst $\min _{\nu}\left(\min _{\alpha, \beta}(\dot{Q})\right)$ is computed numerically by scanning through the possible true anomaly $\nu$ values to find the maximum and minimum $\dot{Q}$ for the particular osculating orbit. The authors note that an alternative approach first found in Ref. 22 can be used which now avoids any numerical derivations thanks to recent work in Ref. 42.

As it can be seen, the inclusion of the second term in Eq. (6) may play an important role in determining the sign of $\dot{Q}$ and, therefore, the stability of the Lyapunov controller. However, its inclusion is often overlooked in existing work in the literature that aims at improving the optimality of Lyapunov controllers. $^{25}$

The following section explains how analytical expressions for the Jacobian matrix of the state-dependent weights can be derived using available information from the actor neural network. The product of this investigation is a state-dependent Lyapunov controller that enforces stability throughout the whole trajectory transfer.

\subsection{Actor Network}

The structure of the actor network is shown in Fig. 1. The actor network maps between input state $\boldsymbol{X}$ and weights $\boldsymbol{W}$, in addition to the effectivity threshold $\eta^{\text {thresh }}$. Actor networks are a common component of RL techniques and their importance is discussed in the subsequent section.

This structure enables us to retain the information on $\partial \boldsymbol{W} / \partial \boldsymbol{X}$ analytically, providing access to the Jacobian matrix of the weights throughout the transfer, and is determined using the chain rule as:

$$
\frac{\partial W_{i}}{\partial X_{j}}=\frac{\partial W_{i}}{\partial \bar{W}_{m}} \frac{\partial \bar{W}_{m}}{\partial \hat{W}_{n}} \frac{\partial \hat{W}_{n}}{\partial X_{j}}
$$

As Fig. 1 shows, the input vector $\boldsymbol{X}$ is first projected onto a grid of radial basis functions (RBFs), which act as features for the network. Each has a centre at a fixed and unchanging location $\boldsymbol{c}_{d}=\left[a_{d}, e_{d}, i_{d}, \Omega_{d}, \omega_{d}\right]^{\mathrm{T}}$. There are $D$ features in the neural network, each with its own centre.

$$
\boldsymbol{C}_{\boldsymbol{X}}=\left[\boldsymbol{c}_{1}, \boldsymbol{c}_{2}, \boldsymbol{c}_{3}, \ldots, \boldsymbol{c}_{D}\right]^{\mathrm{T}}
$$

The basis functions can be written as $\boldsymbol{\psi}=\left[\psi_{1}, \psi_{2}, \psi_{3}, \cdots, \psi_{D}\right]^{\mathrm{T}}$ with scalar components and derivatives with respect to state $\partial \boldsymbol{\psi}(\boldsymbol{X}) / \partial \boldsymbol{X}$ given by: 


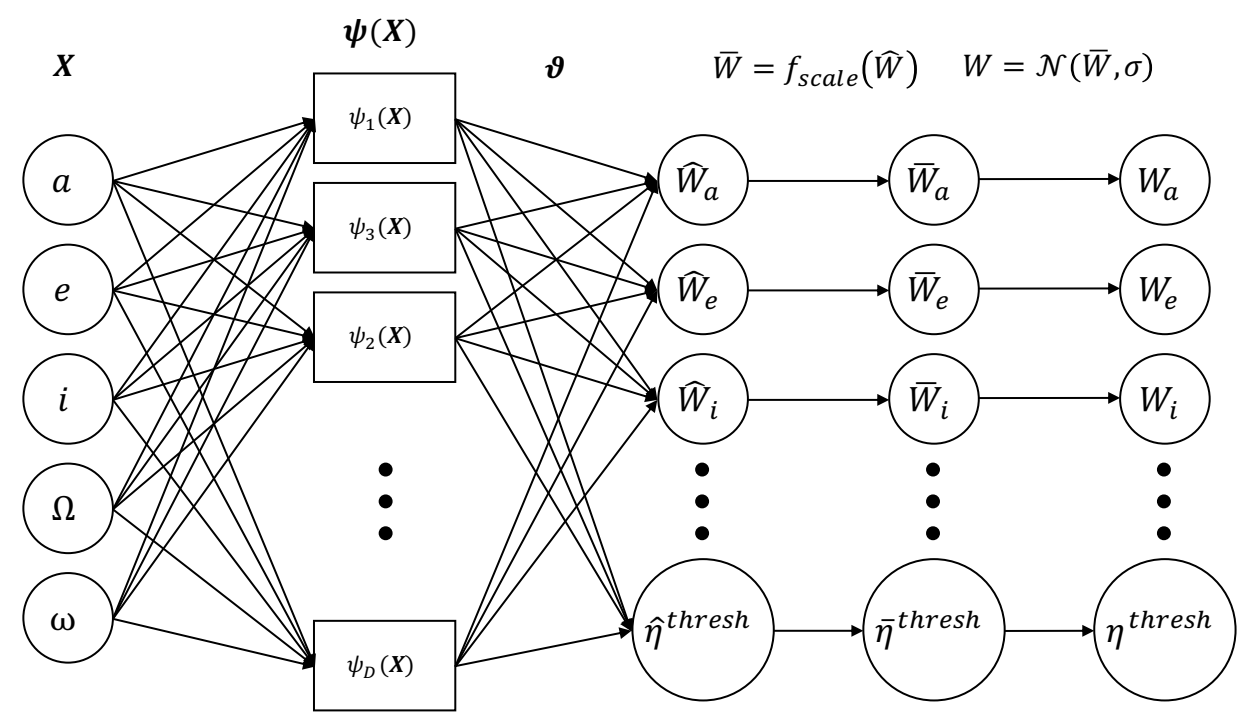

Figure 1: Structure of the actor network. It maps from inputs $\boldsymbol{X}$ to outputs weights $\boldsymbol{W}$ and effectivity $\eta^{\text {thresh }}$. Only $\boldsymbol{W}$ affect the control direction, whilst $\eta^{\text {thresh }}$ determines whether the engine is on or off and can be neglected when calculating the Jacobian.

$$
\begin{aligned}
& \psi_{d}(\boldsymbol{X})=\exp \left(-\frac{1}{2} \frac{\left\|\boldsymbol{X}-\boldsymbol{c}_{d}\right\|^{2}}{\sigma_{\text {net }}^{2}}\right), \\
& \frac{\partial \psi_{d}(\boldsymbol{X})}{\partial \boldsymbol{X}}=\psi_{d}(\boldsymbol{X}) \frac{-\left(\boldsymbol{X}-\boldsymbol{c}_{d}\right)^{\mathrm{T}}}{\sigma_{\text {net }}^{2}}
\end{aligned}
$$

where $\sigma_{\text {net }}$ is the standard deviation of each RBF. The $\boldsymbol{\psi}$ vector is multiplied by a $D \times 6$ matrix of parameters $\theta$ to give the first in a sequence of outputs which will result in the weights vector $\boldsymbol{W}$.

$$
\begin{gathered}
\hat{\boldsymbol{W}}=\theta^{\mathrm{T}} \boldsymbol{\psi}(\boldsymbol{X}) \\
\frac{\partial \hat{\boldsymbol{W}}}{\partial \boldsymbol{X}}=\theta^{\mathrm{T}}\left(\frac{\partial \boldsymbol{\psi}(\boldsymbol{X})}{\partial \boldsymbol{X}}\right)
\end{gathered}
$$

The output is then bounded between 0 and 1 using the hyperbolic tangent function, and this derivative is also readily obtained:

$$
\bar{W}_{i}=f_{\text {scale }}\left(\hat{W}_{i}\right)=\frac{1}{2}\left(\tanh \left(\beta_{f} \hat{W}_{i}\right)+1\right),
$$




$$
\frac{\partial \bar{W}_{i}}{\partial \hat{W}_{j}}= \begin{cases}\frac{\beta_{f}}{2}\left(\operatorname{sech}^{2}\left(\beta_{f} \hat{W}_{j}\right)\right) & \text { if } i=j \\ 0 & \text { if } i \neq j,\end{cases}
$$

where $\beta_{f}$ is a scaling factor that can affect sensitivity of $\theta$ on $\boldsymbol{W}$, here set to $2 \pi$. A stochastic contribution with standard deviation $\sigma$ is used during training to allow the agent to explore the environment. This is switched off during validation/deployment. As this is symmetric about its mean value, the derivative is the identity:

$$
W_{i} \in \mathcal{N}\left(\bar{W}_{i}, \sigma\right) \quad \text { with } \quad \frac{\partial \boldsymbol{W}}{\partial \overline{\boldsymbol{W}}}=\mathbf{1}
$$

Note that as $0 \leq \boldsymbol{W} \leq 1$ issues arises when $W_{i}=\mathcal{N}\left(\bar{W}_{i}, \sigma\right)<0$ or $>1$. We deal with this by re-sampling the distribution until $0 \leq W_{i} \leq 1$.

Combining these contributions, the Jacobian is given by:

$$
\begin{aligned}
\frac{\partial W_{i}}{\partial X_{j}} & =\frac{\partial W_{i}}{\partial \bar{W}_{m}} \frac{\partial \bar{W}_{m}}{\partial \hat{W}_{n}} \frac{\partial \hat{W}_{n}}{\partial X_{j}} \\
& =\frac{\beta_{f} \operatorname{sech}^{2}\left(\beta_{f} \hat{W}_{i}\right)}{2} \sum_{k} \theta_{k i}\left(\frac{\partial \psi_{k}(\boldsymbol{X})}{\partial X_{j}}\right) .
\end{aligned}
$$

This expression for the Jacobian was validated in MATLAB using both a finite difference approach, where small changes in state are reflected in the weights, and a simplified lower-dimension problem.

\subsection{Reinforcement Learning}

To use the actor network, the parameters $\theta$ need to be determined. This is done using a RL algorithm. Critical to the understanding of RL are the agent, policy and reward. Given the state of the environment, the policy will determine what action the agent takes. The agent explores the environment and rewards provide feedback for a given action during the training process. ${ }^{43}$ Hence, the formulation and structure of the reward (or cost) function is critical. During training, an agent will take actions based on an untrained policy, and over time this policy will improve until the optimal policy is learnt. This maps states to actions to maximise the total reward (or minimise the total cost) for the environment. 
More formally, RL problems are usually posed within a Markov decision process (MDP), as sequence of states $x_{i}$, actions $a_{i}$ and a transition dynamics distribution with conditional density $p\left(x_{i+1} \mid x_{i}, a_{i}\right)$, which represents the dynamic relationship between states, given action $a_{i}$ and a cost function. The agent (in our case the spacecraft) interacts with the environment using a parametrised policy $\pi_{\theta}(a \mid x)$ that defines the action taken $a \sim \pi_{\theta}(a \mid x)$. As it interacts with the MDP it collects observations $x_{i}$ and $\operatorname{costs} c\left(x_{i}, a_{i}\right)$ based on the actions taken. For the sequence of states, the discounted cost-to-go $C_{i}$ is often used, which is a discounted sum of the cost $c_{i}=c\left(x_{i}, a_{i}\right)$ at each remaining state along the sequence with discount factor $\gamma \in(0,1]$, and is written as:

$$
C_{i}=\sum_{j=i}^{\text {end }} \gamma^{j-i} c_{j} .
$$

The goal of the agent is to minimise the objective $J\left(\pi_{\theta}\right)=\mathbb{E}\left[C_{0} \mid \pi\right]$ by finding the optimal policy $\pi_{\theta}^{\star}$, which arises from Bellman's equations ${ }^{43}$ and optimises the value function. There are different ways of defining the value function. The state value function $V^{\pi}(x)$ is defined as:

$$
V^{\pi}(x)=\mathbb{E}\left[\sum_{j=0}^{\text {end }} \gamma^{j} c_{j+1} \mid x_{0}=x, \pi\right]
$$

which depends only on the state and assumes the policy $\pi_{\theta}$ is followed starting from this state. The state-action value function $Q^{\pi}(x, a)$ instead is defined as:

$$
Q^{\pi}(x, a)=\mathbb{E}\left[\sum_{j=0}^{\text {end }} \gamma^{j} c_{j+1} \mid x_{0}=x, a_{0}=a, \pi\right]
$$

and depends both on state and action. However, instead of assuming the action $a$ is generated by the policy $\pi_{\theta}$, the action chosen is a free variable. ${ }^{44}$ Once the transition to the next state has been made, the policy $\pi$ determines the subsequent actions $a$. The two value functions can be used to define a quantity called the advantage function:

$$
A^{\pi}(x, a)=Q^{\pi}(x, a)-V^{\pi}(x)
$$

which uses the state value function as a state-dependent baseline to estimate how much better an action is with respect to the expected optimal action under the current policy and is used in general to increase the learning performance. 


\subsubsection{Policy Gradient}

For a continuous state and action space, the most popular RL algorithms are policy gradient algorithms. ${ }^{45,46}$ These use the value function to adjust parameters $\theta$ of a policy $\pi_{\theta}$ in the opposite direction of the gradient of the objective function:

$$
\nabla_{\theta} J\left(\pi_{\theta}\right)=\mathbb{E}\left[\nabla_{\theta} \log \pi_{\theta}(a \mid x) A^{\pi}(x, a)\right] .
$$

Hence, the update is given by:

$$
\theta_{k+1}=\theta_{k}-\alpha_{k} \nabla_{\theta} J_{k}\left(\pi_{\theta}\right),
$$

Actor-critic (AC) algorithms are based on these policy gradient where there are two main components. A critic evaluates the quality of a given action by approximating the value function using a neural network. The actor learns the policy and maps the state to the action the agent should take, often also using a neural network. For more information, Grondman et al. $2012^{44}$ provide a good survey of AC approaches. Normally, AC algorithms use conventional neural networks to represent both the policy and value functions, and use back-propagation to iteratively learn their weights. This is a multiple step process, and is the brain behind the learning process. Extreme Learning Machines (ELMs) ${ }^{47}$ are instead single-layer feed-forward networks (SLFNs) that remove the need for back-propagation, significantly increasing the learning process speed. They assume any continuous target function $f(x)$ can be approximated by a SLFN with a set of $L$ random hidden nodes, activation functions $h(x)$ and learnt parameters $\beta$ as $f_{L}(x)=\sum_{j=1}^{L} \beta_{j} h_{j}(x) .{ }^{47}$ It has been shown that it is both feasible and efficient to use an ELM critic in an $\mathrm{AC}$ algorithm, and the training time for ELM has little impact on the total training time. ${ }^{34}$ In this fashion we can use them successfully here.

\subsubsection{Update}

For a batch of episodes $N$ each with $T$ time-steps, we can write the update as:

$$
\nabla_{\theta} J\left(\pi_{\theta}\right) \approx \frac{1}{N} \sum_{n=1}^{N} \sum_{i=1}^{T} \nabla_{\theta} \log \pi_{\theta}\left(a_{n, i} \mid x_{n, i}\right) \hat{A}^{\pi}\left(x_{n, i}, a_{n, i}\right),
$$

which uses the approximate advantage function

$$
\begin{aligned}
\hat{A}^{\pi}\left(x_{n, i}, a_{n, i}\right) & =\hat{Q}^{\pi}\left(x_{n, i}, a_{n, i}\right)-\hat{V}^{\pi}\left(x_{n, i}\right) \\
& =c\left(x_{n, i}, a_{n, i}\right)+\gamma \hat{V}^{\pi}\left(x_{n, i+1}\right)-\hat{V}^{\pi}\left(x_{n, i}\right) .
\end{aligned}
$$


Here $\hat{V}^{\pi}\left(x_{i}\right)$ indicates the expected cost-to-go of the current state - its value - and $\hat{V}^{\pi}\left(x_{i+1}\right)$ the expected cost-to-go of the subsequent state. Both are approximate given they are learnt by the critic network. The difference between the two is the expected cost of the current state $x_{i}$. Hence, the advantage function reflects how much better the action $a_{i}$ taken in state $x_{i}$ is with respect to the expected action, which can be determined by having multiple trajectories within the same batch during the learning process.

In order to compute the gradient logarithm of the policy, we have to recall the actor network specifically, where the actions $a_{i}$ are given by $\boldsymbol{W}=$ $\left[W_{a}, W_{e}, W_{i}, W_{\Omega}, W_{\omega}, \eta^{\text {thresh }}\right]^{\mathrm{T}}$ and the states $x_{i}$ by the COEs $\boldsymbol{X}$. The stochastic policy means the output weights are given by $W_{i} \in \mathcal{N}\left(\bar{W}_{i}, \sigma\right)$. Hence, we can write

$$
\begin{gathered}
\pi_{\theta} \propto \exp \left(-\frac{1}{2} \frac{(\boldsymbol{W}-\overline{\boldsymbol{W}}(X, \theta))^{2}}{\sigma^{2}}\right), \\
\nabla_{\theta} \log \pi_{\theta}=\frac{\nabla_{\theta} \pi_{\theta}}{\pi_{\theta}}=\frac{(\boldsymbol{W}-\overline{\boldsymbol{W}}(X, \theta))}{\sigma^{2}} \nabla_{\theta} \overline{\boldsymbol{W}}(X, \theta)
\end{gathered}
$$

This derivative is available from the actor network thanks to Eqs. (12) and (15).

\subsection{3. $P P O$}

An RL update strategy based on Proximal Policy Optimisation (PPO) ${ }^{48}$ is used to increase the algorithm's overall performance both in terms of optimally and robustness. This is an AC on-policy algorithm which clips the objective function to remove incentives for the new policy to get too far away from the old policy. In other words it ensures the update size is within a trusted region, attempting to prevent accidentally bad updates. PPO is a simpler and more flexible alternative to Trust-Region Policy Optimisation (TRPO) and can achieve the same high performance. ${ }^{49}$ The objective $J\left(\pi_{\theta}\right)$ is written in terms of a probability ratio $R(\theta)$ between the current and new policies:

$$
R(\theta)=\frac{\pi_{\theta}(a \mid x)}{\pi_{\theta_{k}}(a \mid x)}
$$

$\pi_{\theta}$ represents the latest policy which we are accessing using the current batch. $\pi_{\theta_{k}}$ is the active policy at learning iteration $k$ and is used to generate the batch of trajectories. Using the clipped-PPO approach, the gradient of the

clipped objective is given by two slightly different equations, depending on 
whether the advantage function is positive or negative. For $A^{\pi} \geq 0$,

$$
\begin{aligned}
& \nabla_{\theta} J(\theta)^{\mathrm{CLIP}}=\mathbb{E}\left[\nabla _ { \theta } \operatorname { l o g } \pi _ { \theta } ( a | x ) \operatorname { m i n } \left(R(\theta) A^{\pi}(x, a),\right.\right. \\
&\left.\left.\operatorname{clip}(R(\theta), 1+\epsilon) A^{\pi}(x, a)\right)\right],
\end{aligned}
$$

and for $A^{\pi}<0$ :

$$
\begin{aligned}
\nabla_{\theta} J(\theta)^{\text {CLIP }}= & \mathbb{E}\left[\nabla _ { \theta } \operatorname { l o g } \pi _ { \theta } ( a | x ) \operatorname { m a x } \left(R(\theta) A^{\pi}(x, a),\right.\right. \\
& \left.\left.\operatorname{clip}(R(\theta), 1-\epsilon) A^{\pi}(x, a)\right)\right],
\end{aligned}
$$

where $\epsilon$ is a hyperparameter which determines how far the new policy can deviate from the old.

\section{Implementation}

\subsection{RL Algorithm}

During training two sets of parameters are used: the active $\theta_{k}$ and the newly updated $\theta$. A batch of $N$ trajectories $\tau$ are generated using the stochastic policy $\pi_{\theta_{k}}$. In addition, two deterministic trajectories $\tau\left(\theta_{k}\right)$ and $\tau(\theta)$ are computed. See Fig. 2 for a visual interpretation. Using the costs associated with stochastic trajectories $\tau\left(\pi_{\theta_{k}}\right)$ we can iteratively update $\theta$. Conventionally the updates on $\theta$ and $\theta_{k}$ occur after a predetermined mini-batch size. This limitation can result in a bad update and inevitably make it difficult for the algorithm to converge to an optimal solution. In this work, the update $\theta \leftarrow \theta-\alpha \nabla_{\theta} J(\theta)$ occurs every iteration (i.e., after a batch of $N$ trajectories). However, the update $\theta_{k} \leftarrow \theta$ only occurs if $\tau(\theta)$ outperforms $\tau\left(\theta_{k}\right)$ (namely if $C(\tau(\theta))<C\left(\tau\left(\theta_{k}\right)\right)$ ). An algorithmic pseudocode is show in Algorithm 1.

Each trajectory is divided into fixed time-intervals ensuring the cost associated with each state-action pair is not determined by the time interval. At the start of an interval, the actor network is called with the current state to determine $\boldsymbol{W}$ and $\partial \boldsymbol{W} / \partial \boldsymbol{X}$, which are then kept fixed for the following time-interval. There is a trade-off here: on one hand a smaller time-step allows for more frequent variation of the weights, potentially improving the response by allowing rapid changes in behaviour. However, it also increases the complexity of the learning process. As such, an interval of 0.25 days was found to be most suitable during training. During validation and/or deployment the actor network can be called at the frequency required by the integrator (i.e., embedded) or the satellite operator and, as such, the weights are free to vary at a faster rate. A different transfer problem, particularly one 


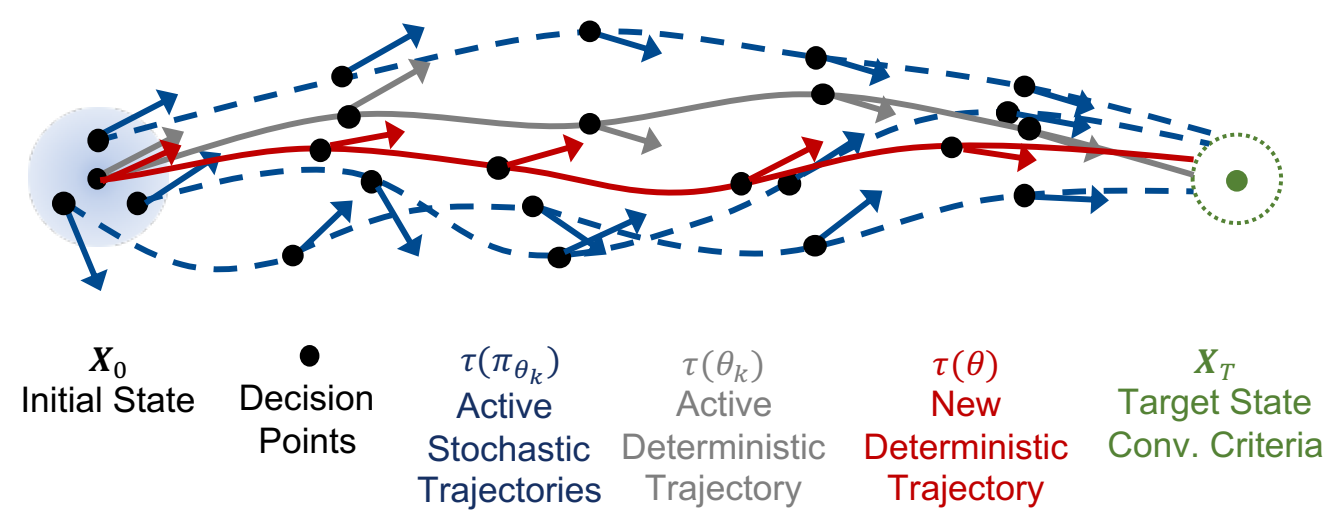

Figure 2: Diagram indicating the different trajectories computed during one learning iteration $k$. Although the control vector is computed at each integration step, the RL decisions are only taken every 0.25 days, indicated by the decision points.

where the expected time-of-flight is much shorter, might require a different time-interval to be selected.

Table 1 gives a summary of the parameters used. 1500 neurons was found to be a suitable number for the critic network and also aligns with a common rule of thumb of $1 / 10$ th of samples used. ${ }^{36}$ One of the major challenges in reinforcement learning algorithms is the exploration-vs-exploitation dilemma. Here this is encapsulated in the parameter $\sigma$, which governs the size of the Gaussian noise added to $\boldsymbol{W}$ at each decision-step during the training process. As $0 \leq \boldsymbol{W} \leq 1$, a nominal value of $\sigma=0.03$ allows the algorithm to explore approximately $10 \%$ of the action space at any given moment. Using a constant value works very well and has achieved satisfactory results in previous work. ${ }^{38,39}$ However, improved results can be obtained when using an adaptive approach. Hence, starting with $\sigma=0.1$, we explore until 50 consecutive iterations occur without improvement, then $\sigma$ is reduced to 0.03. Again if 50 iterations occur without improvement then $\sigma$ is reduced for a second time to 0.01 . If this cycle repeats twice without any improvement then convergence is declared and the algorithm terminates. A fixed learning rate is used throughout. We note that this does not guarantee convergence of the algorithm, however, this is found to work well in many environments and prevents early convergence. ${ }^{43}$

One issue that arises is how to deal with boundaries on the action space. For the Q-law implementation it is necessary to bound $W \geq 0$ and given it 
is normalised we prefer to add the additional constraint $W \leq 1$. As such, we need to transform an infinite space in $\theta$ to a finite one using $f_{\text {scale }}$ in the network architecture. However, taking this output and adding a stochastic element can result in $W$ outside the desired range. Hence, the stochastic noise is generated using $\sigma$ unless $W<3 \sigma$ or $(1-W)<3 \sigma$, in which case $\sigma \leftarrow W / 3$ or $(1-W) / 3$ respectively. These distributions are re-sampled until $0<W<1$.

Due to the feedback nature of the Lyapunov control laws and the fixed magnitude of the control, defining convergence to the target orbit is required. In the past a maximum residual on the orbital elements was used, however, here a more flexible criteria based on the expected time-to-go is implemented instead. The ratio $\delta\left(X, X_{T}\right) / \max _{\nu}(\dot{X})$ gives a measure of the time-to-go and our simulations show this reduces the likelihood of chattering compared to orbital element residuals.

\subsection{Actor-Network Architecture}

As discussed earlier, the actor network governs the relationship between the state and action taken. The authors stress the non-negligible effect the architecture has on the performance of the subsequent implementation. The most influential are the type of basis function $\boldsymbol{\psi}$, the number of neurons (functions) $D$ and the overlap between activation functions $\sigma_{\text {net }}$. The activation functions are evenly spaced over a grid of orbital elements. As such, it is easier to refer to the number of functions per orbital element, $N_{X}$. Two types of activation function are considered, radial basis functions:

$$
\psi_{\mathrm{RBF}}(\boldsymbol{X})=\frac{1}{\prod_{X}\left(N_{X}-1\right)} \frac{1}{\sqrt{2 \pi \sigma_{\text {net }}^{2}}} \exp \left(-\frac{1}{2} \frac{\left\|\boldsymbol{X}-\boldsymbol{c}_{d}\right\|^{2}}{\sigma_{\text {net }}^{2}}\right),
$$

and triangular functions

$$
\psi_{\mathrm{TRI}}(\boldsymbol{X})=\frac{\left(\left\|\boldsymbol{X}-\boldsymbol{c}_{d}\right\|<\sigma_{\mathrm{net}}\right)}{\prod_{X}\left(N_{X}-1\right)} \frac{1}{\sigma_{\mathrm{net}}}\left(1-\frac{\left\|\boldsymbol{X}-\boldsymbol{c}_{d}\right\|}{\sigma_{\mathrm{net}}}\right) .
$$

As seen in Fig. 1, the behaviour of $\boldsymbol{W}$ is governed by $\theta^{\mathrm{T}} \boldsymbol{\psi}$. In order to ensure the state-dependent behaviour is dominated by the parameters to be learnt, $\theta, \sigma_{\text {net }}$ is calculated to minimise the $L 2$-norm between 1 and $\sum_{N_{X}} \psi$, using an approach similar to Ref. 50. This gives much better performance than using $\sigma_{\text {net }}=1 /\left(N_{X}-1\right)$ or using the full-width half-maximum as $\sigma_{\text {net }}$. 
In the triangular-network case, an integer $\sigma_{\text {net }}$ is required, thus, for consistent comparison this value is simply rounded to the closest non-zero integer.

Inputs and outputs are normalised in the network using the range of orbital elements explored by the classical Q-law. If $\left[a_{\text {class }}(t), e_{\text {class }}(t), i_{\text {class }}(t)\right]$ is the full set of states visited by the classical Q-law, the ranges are given by

$$
\begin{aligned}
a_{\min } & =0.75 * \min _{t}\left(a_{\text {class }}(t)\right), \\
a_{\max } & =1.25 * \max _{t}\left(a_{\text {class }}(t)\right), \\
e_{\min } & =\max \left(\min _{t}\left(e_{\text {class }}(t)\right)-0.2,0\right), \\
e_{\max } & =\min \left(\max _{t}\left(e_{\text {class }}(t)\right)+0.2,1\right), \\
i_{\min } & =0.75 * \min _{t}\left(i_{\text {class }}(t)\right), \\
i_{\max } & =1.25 * \max _{t}\left(i_{\text {class }}(t)\right) .
\end{aligned}
$$

This ensures resources aren't wasted on states that the controller is unlikely to visit. However, in many transfer cases, the initial and target values might be the same. We get around this by introducing the $\pm 25 \%$ margin. If one of the edge neurons $C_{X}=X_{T}$ then chattering is observed whilst trying to converge to the target orbit. This is due to the strong influence of the individual $\theta$ parameter associated to $C_{X}$. This is prevented by defining the network based on Eqs. (33) and adding an additional neuron at either end of the network to ensure the observed behaviour occurs inside the network. These additional neurons are added before training starts and can be nonphysical, e.g., centred on a negative eccentricity values. A useful byproduct of this is that any edge effects from the sum over $\psi_{d}$ and their derivative can be reduced. Note, the state of the spacecraft remains physical throughout the learning process.

Figure 3 shows a 1-dimensional representation of these functions and their derivatives when $N_{X}=5$. The individual basis functions are shown, along with their sum for $\theta=1$. By adding an additional neuron outside the domain of interest, we can see the edge effects are reduced within the $0<x<1$ domain. Note in the RBF case there is a non-negligible contribution from $\partial \boldsymbol{\psi} / \partial \boldsymbol{X}$ even when $\theta=1$. As such, when including the Jacobian term in the control, it is not fully determined by $\theta$. This was one of the motivations in exploring the triangular network, where $\partial \boldsymbol{\psi} / \partial \boldsymbol{X}$ is fully determined by the parameters $\theta$. 

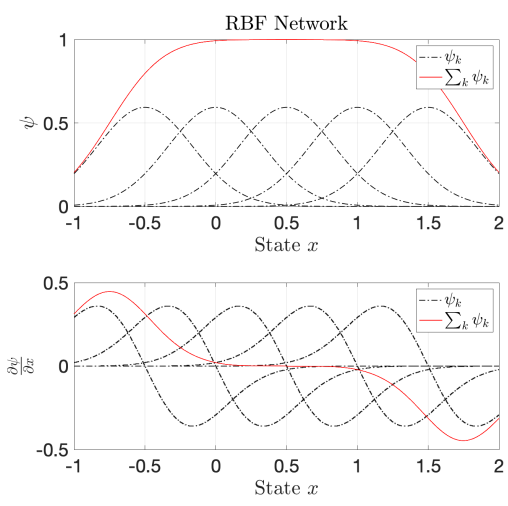

(a) Radial Basis Function Network
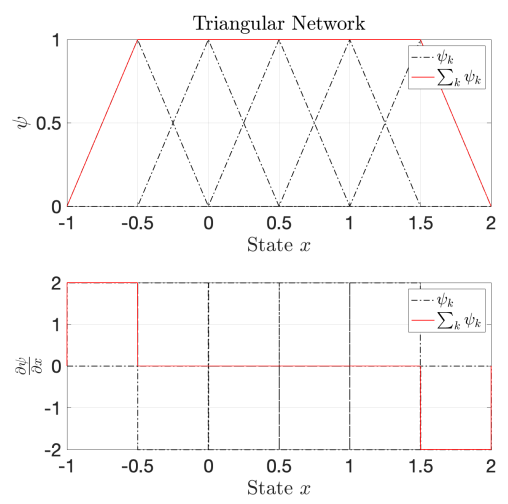

(b) Triangular Basis Function Network

Figure 3: Actor Network architectures visualised in 1-dimension. They show the sum of the basis functions and their derivative with respect to the normalised state input.

Table 1: Table of parameters for RL Q-law implementation. Standard Q-law parameters from Ref. 10 are denoted with petro.

\begin{tabular}{lclccc}
\hline Parameter & Value & Parameter & Value & Parameter & Value \\
\hline \hline Time-interval, $\Delta t$ & 0.25 days & $\gamma$ & 1 & $\sigma_{\text {net }}$ & L2-norm Optimised $^{50}$ \\
Learning rate, $\alpha$ & $2 \mathrm{E}-2,2 \mathrm{E}-3$ & $N_{\text {batch }}$ & $24(22+2)$ & Critic activation & sigmoid \\
$\sigma$ & $0.1,0.03,0.01$ & Grid spacing & 5 & $\#$ critic neurons & 1,500 \\
$\sigma$ interval & 50 iterations & $\#$ actor neurons & 125 & $\epsilon$ & 0.2 \\
$r_{\text {petro }}^{\text {p-min }}$ & 2 & $k_{\text {petro }}$ & 100 & $b_{\text {petro }}$ & 0.01 \\
$r_{\text {petro }}$ & $6,578 \mathrm{~km}$ & $m_{\text {petro }}$ & 3 & $n_{\text {petro }}$ & 4 \\
\hline
\end{tabular}

\section{Results}

Low-thrust time-optimal and mass-optimal transfers from GTO-GEO and LEO-GEO are considered in Keplerian dynamics. The goal is to compare the performance of the RL Q-law and RL Q-Jacobian state-dependent controllers with the literature, constant and time-dependent Q-law approaches. As such, there are 3 distinct benchmark cases. The first is the classical Q-law where we select $W=1$ arbitrarily. However, as many authors have found before, tuning these weights can significantly improve the performance. ${ }^{22,21,25}$ Thus, a particle swarm optimiser (PSO) is used to select more optimal $W$ that remain fixed throughout the transfer (known as "PSO fixed"). Finally, a "PSO spline" approach is used to make the weights time-dependent. The transfer is divided into 3 time-intervals where coefficients are determined by the PSO. Using these coefficients, cubic splines are used to interpolate the value of $W$ at any time, thereby parameterising the weights as piece-wise cubic polyno- 
mials over a grid of time-intervals. This provides a suitable benchmark for the RL Q-law approach, as both PSO approaches use Eq. (5). We don't expect the RL control, with its state-dependent weights, to outperform the PSO spline for a deterministic state. The interested reader can refer to plots on the PSO results in Appendix A.

For the RL Q-law and Q-Jacobian approaches, defining the cost function for a particular objective is key. In the time-optimal case, the cost function is simply the final time-of-flight $t$ in days. If the trajectory doesn't converge within the allowed maximum integration time, then a penalty term on the estimated time-to-go is used, $t_{\text {res }}$. Using $\Delta t_{\text {conv }}$ to denote the convergence criteria, the cost function can be written as:

$$
c^{\mathrm{time}}= \begin{cases}t, & t_{\mathrm{res}} \leq \Delta t_{\mathrm{conv}} \\ t+5 t_{\mathrm{res}}, & t_{\mathrm{res}}>\Delta t_{\mathrm{conv}}\end{cases}
$$

In the mass-optimal case, the cost function is written in-terms of propellant mass used. Note that the mass-optimal trajectories presented here have an upper bound on the time-of-flight, and, as such, a penalty term on the final time is included to enforce this. For convenience and consistency across all cost terms, this time penalty and the time-to-go residual are converted into propellant mass units:

$$
c^{\text {mass }}= \begin{cases}m, & \text { for } t \leq t_{\text {aim }} \\ m+\frac{T}{I_{s p} g_{0}}\left[\left(t-t_{\text {aim }}\right)\right], & \text { and } t_{\text {res }} \leq \Delta t_{\text {conv }} \\ m+\frac{T}{I_{s p} g_{0}}\left[\left(t-t_{\text {aim }}\right)+5 t_{\text {res }}\right], & \text { for } t>t_{\text {aim }} \\ & \text { and } t_{\text {res }} \leq \Delta t_{\text {conv }} \\ & \text { for } t>t_{\text {aim }} \\ & \text { and } t_{\text {res }}>\Delta t_{\mathrm{conv}}\end{cases}
$$

\subsection{GTO-GEO}

Here a GTO-GEO transfer with an inclination change is considered in Keplerian dynamics. The parameters are chosen to compare with Refs. 25 and 51 and were considered by the authors in Refs. 38 and 39, where significant improvements can be seen. The modelled spacecraft has mass $=2000$ $\mathrm{kg}$, thrust $=0.35 \mathrm{~N}$ and Isp $=2000 \mathrm{~s}$, giving an initial thrust-to-mass ratio of $0.000175 \mathrm{~ms}^{-2}$. The initial and target orbits are shown in Table 2. Unlike in previous references, the convergence criteria is now placed on the Q-law 
residual rather than individual elements. A 0.25 day residual is chosen as it provides comparable results with previous criteria and because it is the decision time-step in the RL algorithm. These convergence criteria prevent the Q-law from chattering close to the target orbit.

Table 2: Initial and target orbital elements for a GTO-GEO transfer. The modelled spacecraft has mass $2000 \mathrm{~kg}$, thrust $0.35 \mathrm{~N}$ and Isp $2000 \mathrm{~s}$.

\begin{tabular}{ccccccc}
\hline & $a(\mathrm{~km})$ & $e$ & $i(\mathrm{deg})$ & $\Omega(\mathrm{deg})$ & $\omega(\mathrm{deg})$ & $\nu(\mathrm{deg})$ \\
\hline \hline Initial & $24,505.9$ & 0.725 & 7 & 0.0 & 0.0 & 0.0 \\
Target & $42,165.0$ & $1 \mathrm{e}-5$ & 0 & free & free & free \\
\hline
\end{tabular}

Results are given in Table 3. It is clear from the PSO fixed simulations that the optimality in terms of time-of-flight can be improved compared to the time-optimal classical Q-law by tuning the weights, as many have found before. $^{22,21,25}$ The PSO spline makes the weights time-dependent and for time-optimal transfers should provide an indication of the best possible solution available to the RL approaches. The RL Q-law refers to the Q-law with

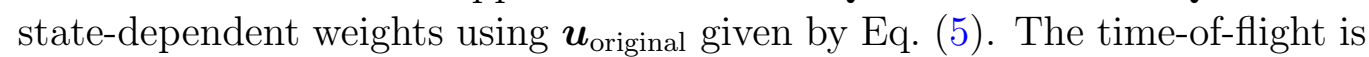
137.14 days. Adding the estimated time-to-go of 0.25 days from the convergence criteria, it matches the result found in Ref. 25. Using $\boldsymbol{u}_{\text {jacobian }}$ gives a time-of-flight of 137.45 days whilst also guaranteeing Lyapunov stability. State-dependent weights result in a minor increase in optimality compared to the optimised constant weight solution. The simulations using the triangular network give very similar results, indicating different network architectures can be accommodated in the algorithm.

For the mass-optimal simulations, the absolute effectivity $\eta_{a}$ was used and a target time-of-flight of 150 days was set. Compared to the time-optimal classical Q-law, the best solution can save $31.05 \mathrm{~kg}(14.0 \%)$ at a cost of 5.65 days (3.9\%). Again, there is little difference between the stable RL QJacobian and RL Q-law. The constant weight solution is $1.13 \mathrm{~kg}$ worse than the best state-dependent one.

Figure 4 shows the RL Q-law and RL Q-Jacobian results for the timeoptimal simulations. Interestingly in Fig. 4a it is clear the inclination needs to be prioritised in order to reach GEO with a faster time-of-flight. Figure 4c indicates the $\boldsymbol{W}$ learnt by the RL algorithm. However, Fig. 4d gives a better indication of which component is dominating the control term. In both cases, the eccentricity is the most influential, followed by the semi-major axis term. 
Table 3: Comparison of state-dependent Q-law performance for GTO-GEO transfer, using a spacecraft with mass $2000 \mathrm{~kg}$, thrust $0.35 \mathrm{~N}$ and Isp 2000s.

\begin{tabular}{llll}
\hline Method & $\begin{array}{l}\text { Time } \\
\text { (days) }\end{array}$ & $\begin{array}{l}\text { Propellant } \\
\text { (kg) }\end{array}$ \\
\hline \hline Time & Time-optimal Classical & 144.03 & 222.06 \\
& Ref. 25 & 137.3 & 211.72 \\
& Ref. 51 & 137.5 & 212.00 \\
& PSO fixed & 137.90 & 212.61 \\
PSO spline & 137.16 & 211.47 \\
RL Q-law (rbf) & 137.14 & 211.44 \\
RL Q-Jacobian (rbf) & 137.45 & 211.93 \\
RL Q-law (tri) & 137.24 & 211.59 \\
RL Q-Jacobian (tri) & 137.90 & 212.62 \\
\hline Mass & 150.00 & 187.97 \\
Ref. 25 & 150.00 & 192.00 \\
Ref. 51 & 149.75 & 192.14 \\
PSO fixed & 149.75 & 190.03 \\
PSO spline & 149.68 & 191.01 \\
RL Q-law (rbf) & 149.73 & 191.82 \\
RL Q-Jacobian (rbf) & 149.90 & 192.33 \\
RL Q-law (tri) & 149.76 & 191.65 \\
\hline
\end{tabular}

Both these have very large changes to undergo. We note the time-history observed in the RL Q-law and RL Q-Jacobian weights shares similarities to the PSO spline approach - see Appendix A.

Figures 4 e and 4 f indicate the $\dot{Q}$ behaviour throughout the transfer. $\dot{Q}_{\boldsymbol{X}}$ and $\dot{Q}_{\boldsymbol{W}}$ refer to the contributions to $\dot{Q}$ from the state $(\partial Q / \partial \boldsymbol{X})$ and weights $(\partial Q / \partial \boldsymbol{W})$, respectively. Figure 4e shows that $\dot{Q}_{\boldsymbol{X}}<0$ throughout the transfer. However, due to the neglected contribution of $\dot{Q}_{\boldsymbol{W}}$, the true $\dot{Q}$ is greater than zero for short but significant periods during the middle of the transfer. It follows that one of the major requirements of Lyapunov control theory is violated, leading to potentially unsafe behaviour. This lack of guaranteed stability could be a major concern to mission operators, but the introduction of the analytical Jacobian removes this issue with little compromise on optimality.

The physical effect of $\dot{Q}>0$ is an increase in the Lyapunov function 
value, increasing the expected time-to-go for the transfer. However, this doesn't always correspond to an increased difference between current and target orbital elements, as the denominator in Eq. 3 needs to be considered. In the RL Q-law case it is possible to generate pathological setups of $W$ which causes $Q$ to increase through the transfer and not converge to the target orbit, although this is very rare during the learning process as non-converging transfers are easily discarded. The addition of the Jacobian removes this issue entirely. The current results are obtained using osculating orbital elements. If averaged dynamics are used, it is likely $\dot{Q}$ would appear less than zero for the duration of the transfer for both $\boldsymbol{u}_{\text {original }}$ and $\boldsymbol{u}_{\text {jacobian }}$, as each violation is less than one orbital period. In the case of $\boldsymbol{u}_{\text {original }}$ that wouldn't be telling the full story and the impact of this might need to be investigated in future work.

In the mass-optimal case, similar plots are given in Fig. 5. Figure 5b shows coast arcs occurring predominantly at the osculating apoapses, matching our intuitive understanding of these kinds of orbital transfers. Again Figs. 5e and 5 f show that $\boldsymbol{u}_{\text {jacobian }}$ ensures $\dot{Q}<0$ throughout.

Figure 6 shows the evolution of $\eta_{a}$ for both the $\boldsymbol{u}_{\text {original }}$ and $\boldsymbol{u}_{\text {jacobian }}$ trajectories. Eq. (7) is used in both cases, but only with the available knowledge of $\dot{Q}$. Although conventionally $0<\eta_{a} \leq 1$, this assumes $\eta_{a}$ and the control $\boldsymbol{u}$ are computed with the full knowledge of $\dot{Q}$. This is the case for $\boldsymbol{u}_{\text {jacobian }}$, as seen in Fig. 6b, however, for $\boldsymbol{u}_{\text {original }}$ a contribution to $\dot{Q}$ is unknown. The effectivity used during the transfer is given by:

$$
\eta_{a}\left(\dot{Q}_{X}\right)=\frac{\min _{\alpha, \beta}\left(\dot{Q}_{X}\right)}{\min _{\nu}\left(\min _{\alpha, \beta}\left(\dot{Q}_{X}\right)\right)} .
$$

Retrospectively, when accounting for the neglected contribution to $\dot{Q}$, the true $\eta_{a}$ appears negative in places, as seen in Fig. 6a. Due to this incomplete information on the most efficient places to thrust, a negative retrospective effectivity means the thrust was actually increasing the value of $Q$ rather than decreasing it as desired. Fortunately, although $\eta_{a}(\dot{Q})<0$ for $1.2 \%$ of the transfer, this occurs exclusively when the engine is off. In fact, there is only $6.7 \%$ of the time when a more efficient direction could be computed, reducing to $5.3 \%$ when the engine is on.

\section{2. $L E O-G E O$}

Here a LEO-GEO transfer with an inclination change is considered in Keplerian dynamics. The parameters are chosen to allow comparison with 
Ref. 14 and similar ones were considered by the authors in Refs. 38 and 39. The modelled spacecraft has mass $=1200 \mathrm{~kg}$, thrust $=0.4017 \mathrm{~N}$ and Isp $=3300 \mathrm{~s}$, giving an initial thrust-to-mass ratio of $0.00033475 \mathrm{~m} \mathrm{~s}^{-2}$, approximately double the previous test case. The initial and target orbits are shown in Table 4. Again a 0.25 day residual is chosen to define convergence to the target orbit.

Table 4: Initial and target orbital elements, and convergence criteria, for a LEO-GEO transfer. The modelled spacecraft has mass $1200 \mathrm{~kg}$, thrust $0.4017 \mathrm{~N}$ and Isp $3300 \mathrm{~s}$.

\begin{tabular}{ccccccc}
\hline & $a(\mathrm{~km})$ & $e$ & $i(\mathrm{deg})$ & $\Omega(\mathrm{deg})$ & $\omega(\mathrm{deg})$ & $\nu(\mathrm{deg})$ \\
\hline \hline Initial & 6927 & $1 \mathrm{e}-5$ & 28.5 & 0.0 & 0.0 & 0.0 \\
Target & 42,164 & $1 \mathrm{e}-5$ & 0 & free & free & free \\
\hline
\end{tabular}

Results are given in Table 5. Using $\boldsymbol{u}_{\text {original }}$, the RL Q-law time-of-flight was 180.38 days. However, using $\boldsymbol{u}_{\text {jacobian }}$, the optimality increased to 179.77 days. It is also important to note that this result betters the time-dependent PSO, where the control is forced to be $\boldsymbol{u}_{\text {original }}$ as information of $(\partial Q / \partial \boldsymbol{W})$ $(\partial \boldsymbol{W} / \partial \boldsymbol{X})$ is not available. A key advantage of the RL approach is that the Jacobian matrix can be calculated analytically from the structure of the actor network.

For the mass-optimal simulations, the absolute effectivity $\eta_{a}$ was used and a target time-of-flight of 200 days was set. Compared to the time-optimal classical Q-law, the best solution saves $26.77 \mathrm{~kg}(12.6 \%)$ at a cost of 1.37 days $(0.7 \%)$, whilst it improves on the constant weight solution by 10.19 $\mathrm{kg}$. Unlike in the GTO-GEO transfer, there is a significant improvement on the fixed PSO results for both time and mass optimal transfers. This clearly demonstrates the advantage of varying the parameters throughout the transfer. Here the RL Q-Jacobian not only enforces stability but significantly increases the optimality of the mass-optimal solution. This can be explained by the effectivity below.

Figures 7 and 8 shows the RL Q-law and RL Q-Jacobian results for the time-optimal and mass-optimal simulations. Again the plots of $\dot{Q}$ indicate a lack of Lyapunov stability in the RL Q-law case, but this is resolved by adding the Jacobian of the state-dependent weights. Intuition on the timehistories observed in the RL Q-law and RL Q-Jacobian weights can be drawn from the PSO spline approach - see Appendix A.

Figure 9 shows the evolution of $\eta_{a}$ for both the $\boldsymbol{u}_{\text {original }}$ and $\boldsymbol{u}_{\text {jacobian }}$ tra- 
Table 5: Comparison of state-dependent Q-law performance for LEO-GEO transfer, using a spacecraft with mass $1200 \mathrm{~kg}$, thrust $0.4017 \mathrm{~N}$ and Isp $3300 \mathrm{~s}$

\begin{tabular}{llll}
\hline Method & $\begin{array}{l}\text { Time } \\
\text { (days) }\end{array}$ & $\begin{array}{l}\text { Propellant } \\
\text { (kg) }\end{array}$ \\
\hline \hline Time & Time-optimal Classical & 198.32 & 212.70 \\
& Ref. 14 & 198.99 & - \\
& PSO fixed & 186.10 & 199.58 \\
PSO spline & 180.68 & 193.77 \\
RL Q-law (rbf) & 180.38 & 193.45 \\
RL Q-Jacobian (rbf) & 179.77 & 192.80 \\
RL Q-law (tri) & 181.07 & 194.20 \\
RL Q-Jacobian (tri) & 180.46 & 193.54 \\
\hline Mass & PSO fixed & 199.67 & 196.52 \\
PSO spline & 199.74 & 189.43 \\
RL Q-law (rbf) & 198.81 & 192.10 \\
RL Q-Jacobian (rbf) & 199.69 & 185.93 \\
RL Q-law (tri) & 199.74 & 190.87 \\
RL Q-Jacobian (tri) & 199.59 & 186.81 \\
\hline
\end{tabular}

jectories. Again Eq. (7) is used in both cases, but only with the available knowledge of $\dot{Q}$, and, as such, the RL Q-law is using Eq. (36). Unlike in the GTO-GEO case where this had minimal effect, here it can explain the discrepancy between the RL Q-law and RL Q-Jacobian solutions. In Fig. 9a, $\eta_{a}(\dot{Q})<0$ occurs $12.0 \%$ of the time, and $11.8 \%$ when the engine is on, suggesting thrusting here is actually ineffective. In addition, there is a more efficient control direction for $20.9 \%$ of the transfer, reducing to $16.0 \%$ when the engine is on.

\subsection{Robustness to Eclipses}

One major test for a low-thrust trajectory design algorithm is its ability to handle discontinuities such as eclipse events, which can be very challenging for conventional trajectory design techniques.

Two approaches are considered. The first takes the agent trained without eclipses and deploys it in the presence of eclipses, and the second involves re-training the algorithm with eclipse effects included. In both cases the existence of eclipse events is not communicated to the agent. Instead, the eclipse event is only computed inside the propagator and results in an inability to 
fire the engine. The presence of eclipse events is indirectly learnt by the agent by observing that particular actions have reduced effect on the trajectory's cost as they occur during eclipse events.

Table 6: Comparison of state-dependent Q-law performance for GTO-GEO and LEO-GEO transfers including eclipse effects. Epoch: $1^{\text {st }}$ January 2022 12:00:00 UTC.

\begin{tabular}{llcccc}
\hline Transfer & Method & \multicolumn{2}{c}{ Trained without Eclipses } & \multicolumn{2}{c}{ Trained with Eclipses } \\
& & ToF (days) & Prop (kg) & ToF (days) & Prop (kg) \\
\hline \hline GTO-GEO & Classical & 151.23 & 224.13 & - & - \\
& RL Q-law (rbf) & 142.25 & 213.39 & 142.04 & 213.30 \\
& RL Q-Jacobian (rbf) & 142.40 & 213.62 & 142.15 & 213.37 \\
& RL Q-law (tri) & 142.33 & 213.45 & 142.63 & 213.74 \\
& RL Q-Jacobian (tri) & 143.27 & 214.54 & 142.79 & 214.10 \\
\hline LEO-GEO & Classical & 227.41 & 205.09 & - & - \\
& RL Q-law (rbf) & 224.88 & 199.62 & 219.86 & 195.72 \\
& RL Q-Jacobian (rbf) & 219.17 & 195.58 & 216.78 & 193.71 \\
& RL Q-law (tri) & 221.93 & 197.53 & 217.36 & 193.98 \\
& RL Q-Jacobian (tri) & 217.28 & 194.76 & 214.98 & 192.47 \\
\hline
\end{tabular}

Table 6 shows the performance of the algorithm in the presence of eclipses. Training with eclipse included results in a 9.19 and 9.08 day improvement on the classical Q-law for the GTO-GEO transfer. In the LEO-GEO case, the time-of-flight increases significantly on the time-optimal solution, from 179.77 days to 214.98 days, owing to the frequent eclipse events in LEO. In addition, when training with eclipse events it becomes possible to gain several extra days by altering the trajectory to remain out of eclipse for longer. Figures 10a and 10b show these trajectories for the RL Q-Jacobian solutions. These results highlight the importance of the state-dependent control over time-dependent control.

\subsection{Robustness to Navigation Errors and Thrust misalignment}

One of the major advantages of Lyapunov-based controllers is their closedloop nature. By design this is maintained in the RL formulation and is investigated here. In order to assess the impact of orbit injection, navigation, and thrust execution errors on the RL Q-law and RL Q-Jacobian, several 1000-sample Monte Carlo simulation were initiated. The errors modelled in our simulations are assumed to be: 
- Orbit injection: zero-mean Gaussian errors with a $1 \sigma=10 \mathrm{~km}$ and $1 \sigma=1 \mathrm{~m} / \mathrm{s}$ spherical standard deviations in the position and velocity components of the spacecraft initial state;

- Knowledge: zero-mean Gaussian errors with $1 \sigma=100 \mathrm{~m}$ (position), and $1 \sigma=10 \mathrm{~cm} / \mathrm{s}$ (velocity) standard deviations;

- Thrust magnitude: random noise added to the nominal thrust value. The stochastic component follows a Gaussian distribution with zeromean, $3 \sigma=5 \%$ the nominal thrust value;

- Thrust direction: uniformly distributed biases of \pm 10 deg in elevation and $\pm 180 \mathrm{deg}$ in the azimuth angles of the thrust vector. These biases are kept constant throughout the transfer and augmented with a zeromean stochastic component of $3 \sigma=2 \mathrm{deg}$ in elevation and $3 \sigma=20 \mathrm{deg}$ in azimuth at each thrust calculation epoch (every 1 minute).

The thrust errors are pessimistic in order to assess the robustness of our approach under extreme circumstances. These errors are added every 1 minute, during which the control direction and magnitude are kept constant. This frequency is selected to cause the most difficulty for the controller. Reducing it reduces both the mean and standard deviation of the resulting Monte Carlo simulations.

Starting from the nominal initial orbit at periapsis, injection errors are added to determine the true initial state of the spacecraft. Navigation errors are simulated next in order to create an estimate for the current state of the spacecraft at the beginning of a "guidance loop". Here, the best estimate of the spacecraft state is passed to the control function for determining the nominal control direction $\boldsymbol{u}$. A $\delta u$ is added to the magnitude and the thrust direction is also modified. Here, a right-handed frame is defined with respect to $\boldsymbol{u}$ and the angular momentum $\boldsymbol{h}$ of the osculating orbit as follows:

$$
\left[\begin{array}{lll}
\hat{\boldsymbol{e}}_{1} & \hat{\boldsymbol{e}}_{2} & \hat{\boldsymbol{e}}_{3}
\end{array}\right]=\left[\begin{array}{lll}
\frac{\boldsymbol{u}}{\|\boldsymbol{u}\|} & \frac{\boldsymbol{h} \times \boldsymbol{u}}{\|\boldsymbol{h} \times \boldsymbol{u}\|} & \frac{\hat{\boldsymbol{e}}_{1} \times \hat{\boldsymbol{e}}_{2}}{\left\|\hat{\boldsymbol{e}}_{1} \times \hat{\boldsymbol{e}}_{2}\right\|}
\end{array}\right] .
$$

The elevation $\phi_{e}$ and azimuth $\phi_{a}$ angles are defined in this local reference frame such that the actual thrust direction is

$$
\boldsymbol{u}^{*}=(\|\boldsymbol{u}\|+\delta u)\left[\begin{array}{c}
\cos \phi_{e} \\
\sin \phi_{e} \cos \phi_{a} \\
\sin \phi_{e} \sin \phi_{a}
\end{array}\right] .
$$


It follows that an error on the elevation angle can have a larger impact on the actual direction of thrust.

Figure 11 shows histograms of the results from these Monte Carlo simulations. It is clear that the optimality offered by the RL approach is retained for both the time and mass-optimal transfers. It highlights the closed-loop nature of this approach, and that it functions away from the nominal trajectory. Even with navigation errors and thruster misalignment, the inherent advantages of using a Lyapunov-based controller are retained. In Table 7 it is also clear that the standard deviation of the runs, when subject to identical errors, is smaller for the RL approaches. Finally, none of the simulated RL approaches under-perform compared to the classical Q-law.

Note that the constant control direction and magnitude might be expected to affect the performance, but the results suggest this has little impact for this time-step. Including these restrictions and uncertainties in the training process could further extend the validity of this approach, but is out of scope for this work.

Table 7: Monte Carlo Simulations with stochastic disturbances for the GTO-GEO and LEO-GEO transfers. Nominal results are shown for comparison.

\begin{tabular}{|c|c|c|c|c|c|c|c|c|}
\hline & Transfer & Method & Nominal & Mean & $\sigma$ & $1^{\text {st }}$ Percentile & Median & $99^{\text {th }}$ Percentile \\
\hline \multirow{7}{*}{$\begin{array}{l}\text { Time } \\
\text { (days) }\end{array}$} & \multirow[t]{3}{*}{ GTO-GEO } & Classical & 144.03 & 144.99 & 2.55 & 141.88 & 144.12 & 152.73 \\
\hline & & RL Q-law & 137.14 & 137.94 & 0.78 & 136.29 & 137.91 & 139.87 \\
\hline & & RL Q-Jacobian & 137.45 & 138.21 & 0.84 & 136.52 & 138.11 & 140.28 \\
\hline & \multirow{3}{*}{ LEO-GEO } & Classical & 198.32 & 198.27 & 1.02 & 196.38 & 198.12 & 200.86 \\
\hline & & RL Q-law & 180.38 & 181.08 & 0.72 & 179.82 & 180.94 & 183.07 \\
\hline & & RL Q-Jacobian & 179.77 & 180.61 & 0.77 & 179.28 & 180.45 & 182.64 \\
\hline & Transfer & Method & Nominal & Mean & $\bar{\sigma} \sigma$ & $1^{\text {st }}$ Percentile & Median & $99^{\text {th }}$ Percentile \\
\hline \multirow{6}{*}{$\begin{array}{l}\text { Mass } \\
(\mathrm{kg})\end{array}$} & \multirow[t]{3}{*}{ GTO-GEO } & Classical & 222.06 & 223.56 & 3.93 & 218.75 & 222.21 & 235.48 \\
\hline & & RL Q-law & 191.01 & 192.01 & 2.32 & 187.97 & 191.49 & 197.56 \\
\hline & & RL Q-Jacobian & 191.82 & 193.82 & 3.12 & 190.20 & 192.62 & 203.89 \\
\hline & \multirow[t]{3}{*}{ LEO-GEO } & Classical & 212.70 & 212.64 & 1.11 & 210.59 & 212.48 & 215.45 \\
\hline & & RL Q-law & 192.10 & 192.77 & 0.78 & 191.36 & 192.64 & 194.83 \\
\hline & & RL Q-Jacobian & 185.93 & 187.02 & 1.01 & 185.40 & 186.81 & 189.32 \\
\hline
\end{tabular}

\section{Conclusion}

This paper elaborates on the optimality of state-dependent Lyapunov controllers that have the potential to enable low-thrust autonomous operations in space. A PPO reinforcement learning approach is used to increase the optimality of these controllers whilst maintaining their stable closed-loop 
nature. Unlike previous work in the literature, we derive a modified control law that accounts for contributions due to the resulting state-weight relationship, thus enforcing asymptotic stability throughout the transfer. The necessary Jacobian is available analytically through an actor neural network, ensuring the system remains closed-loop. Combining a Lyapunov controller with an RL architecture allows one to eliminate the drawbacks from each approach: namely the sub-optimality of Lyapunov controllers and the unknown stability of RL methods.

Investigations for transfers from a geostationary transfer orbit to geostationary orbit (GTO-GEO) and low-Earth orbit to geostationary orbit (LEOGEO) show the control can ensure stability at each state in the transfer. Making the parameters state-dependent significantly increases the optimality of the solutions found for both mass-optimal transfers and the LEO-GEO time-optimal transfer. The demonstrated controller is also robust to uncertainties in state and eclipse effects, retaining a high degree of optimality and demonstrating the closed-loop nature of this approach. This paves the way for future work in higher-fidelity dynamics. Although out-of-scope for this work, the method offers a lot of potential for on-board use. The neural network can be trained on-ground and the trained network implemented onboard to offer increased optimality along with desired stability compared to a classical Lyapunov feedback controller. This closed-loop guidance approach would be able to execute an autonomous transfer, or reconfiguration, in an close-to-optimal manner, with demonstrated robustness to uncertainties in state, thruster errors and eclipse periods.

\section{Acknowledgements}

Harry Holt is funded by Surrey Satellite Technology Limited (SSTL). $\mathrm{HH}$ thanks Peter Senior and Steve Eckersley from SSTL and Chris Bridges from Surrey Space Centre (SSC) for their invaluable discussions and input. In addition, the authors would also like to thank Abolfazl Shirazi for discussions regarding the PSO spline approach.

\section{Appendix A. Particle Swarm Optimised Q-law transfers}

In this Appendix the results for the fixed and spline particle swarm optimised (PSO) Q-law transfers are presented. The weight profiles and contributions to the Q-law are given as a comparison to the RL Q-law and 
RL Q-Jacobian solutions presented in the main paper. For the GTO-GEO transfer, Figs. A.12a and A.12b should be compared with Figs. 4c and 4d respectively; and Figs. A.12c and A.12d with Figs. 5c and 5d. As for the LEO-GEO transfer, Figs. A.13a and A.13b correspond to Figs. 7c and 7d; and Figs. A.13c and A.13d to Figs. 8c and 8d.

\section{References}

[1] D. R. Lev, R. M. Myers, K. M. Lemmer, The Technological and Commercial Expansion of Electric Propulsion in the Past 24 Years, Proceedings of the 35th IEPC (October) (2017) IEPC-2017-242.

[2] Eutelsat, All-electric Eutelsat 172B satellite set to transfer connectivity landscape in Asia-Pacific (2017).

URL https://www.eutelsat.com/en/news/press.html\#/ pressreleases/eutelsats-airbus-built-full-electriceutelsat-172b-satellite-reaches-geostationary-orbit2208095

[3] T. Saiki, J. Matsumoto, O. Mori, J. Kawaguchi, Solar Power Sail Trajectory Design for Jovian Trojan Exploration, Trans. JSASS Aerospace Tech. Japan 16 (5) (2018) 353-359.

[4] T. N. Edelbaum, Propulsion Requirements for Controllable Satellites, ARS Journal 31 (8) (1961) 1079-1089.

[5] J. T. Betts, Practical Methods for Optimal Control and Estimation Using Nonlinear Programming, Society for Industrial and Applied Mathematics, 2010.

[6] B. A. Conway, Spacecraft trajectory optimization, Cambridge University Press, 2010.

[7] A. Shirazi, J. Ceberio, J. A. Lozano, Spacecraft trajectory optimization: A review of models, objectives, approaches and solutions, Progress in Aerospace Sciences 102 (August) (2018) 76-98.

[8] C. A. Kluever, Simple guidance scheme for low-thrust orbit transfers, Journal of Guidance, Control, and Dynamics 21 (6) (1998) 1015-1017. 
[9] A. E. Petropoulos, Simple control laws for low-thrust orbit transfers, AAS/AIAA Astrodynamics Specialists Conference (2003).

[10] A. E. Petropoulos, Refinements to the Q-law for low-thrust orbit transfers, in: Advances in the Astronautical Sciences, Vol. 120, 2005, pp. 963-982.

[11] Y. Gao, X. Li, Optimization of low-thrust many-revolution transfers and Lyapunov-based guidance, Acta Astronautica 66 (1-2) (2010) 117-129.

[12] J. L. Shannon, M. T. Ozimek, J. A. Atchison, M. Christine, Q-law aided Direct Trajectory Optimization for the High-fidelity, Many-revolution Low-thrust Orbit Transfer Problem, in: AAS, no. 19, 2019, p. 448.

[13] H. Schaub, J. L. Junkins, Analytical Mechanics of Space Systems, Fourth Edition, American Institute of Aeronautics and Astronautics, Inc., 2018.

[14] R. Falck, W. Sjauw, D. Smith, Comparison of Low-Thrust control laws for application in planetocentric space, 50th AIAA/ASME/SAE/ASEE Joint Propulsion Conference 2014 (2014) 1-14.

[15] B. E. Joseph, Lyapunov Feedback Control in Equinoctial Elements Applied to Low Thrust Control of Elliptical Orbit Constellations, Msc thesis, Massachusetts Institute of Technology (2006).

[16] B. J. Naasz, Classical Element Feedback Control for Spacecraft Orbital Maneuvers, Msc thesis, Virginia Polytechnic Institute and State University (2002).

[17] C. Maddock, M. Vasile, Extension of the proximity-quotient control law for low-thrust propulsion, International Astronautical Federation - 59th International Astronautical Congress 2008, IAC 20088 (July) (2008) 4901-4915.

[18] N. Baresi, L. Dell'Elce, J. Cardoso dos Santos, Y. Kawakatsu, Orbit maintenance of quasi-satellite trajectories via mean relative orbit elements, Iac-2018 (2018) 1-11.

[19] R. Epenoy, D. Pérez-Palau, Lyapunov-based low-energy low-thrust transfers to the Moon, Acta Astronautica 162 (June) (2019) 87-97. 
[20] M. Pontani, M. Pustorino, Nonlinear Earth orbit control using lowthrust propulsion, Acta Astronautica 179 (2021) 296-310.

[21] S. Lee, A. Petropoulos, P. von Allmen, Low-thrust Orbit Transfer Optimization with Refined Q-law and Multi-objective Genetic Algorithm, Advances In The Astronautical Sciences (2005).

[22] G. Varga, J. M. Sánchez Pérez, Many-Revolution Low-Thrust Orbit Transfer Computation Using Equinoctial Q-Law Including J2 and Eclipse Effects, Icatt 2016 (2016) 2463-2481.

[23] A. E. Petropoulos, Z. B. Tarzi, G. Lantoine, T. Dargent, R. Epenoy, Techniques for designing many-revolution, electric-propulsion trajectories (AAS 14-373) (2014).

[24] D. Izzo, M. Märtens, B. Pan, A Survey on Artificial Intelligence Trends in Spacecraft Guidance Dynamics and Control, arXiv preprint arXiv:1812.02948 (2018).

[25] D. L. Yang, B. Xu, L. Zhang, Optimal low-thrust spiral trajectories using Lyapunov-based guidance, Acta Astronautica 126 (2016) 275-285.

[26] B. Gaudet, R. Linares, R. Furfaro, Deep Reinforcement Learning for Six Degree-of-Freedom Planetary Powered Descent and Landing (2018). arXiv: 1810.08719.

[27] A. Scorsoglio, R. Furfaro, R. Linares, B. Gaudet, Image-based Deep Reinforcement Learning for Autonomous Lunar Landing, in: AIAA Scitech 2020 Forum, 2020.

[28] B. Gaudet, R. Furfaro, Robust Spacecraft Hovering Near Small Bodies in Environments with Unknown Dynamics Using Reinforcement Learning, AIAA/AAS Astrodynamics Specialist Conference (May 2018) (2012).

[29] B. Gaudet, R. Linares, R. Furfaro, Six Degree-of-Freedom Hovering using LIDAR Altimetry via Reinforcement Meta-Learning (2019). arXiv: 1911.08553.

[30] D. Miller, R. Linares, Low-Thrust Optimal Control Via Reinforcement Learning, in: AAS, no. February, 2019, pp. 1-20. 
[31] N. B. LaFarge, D. Miller, K. C. Howell, R. Linares, Guidance for ClosedLoop Transfers using Reinforcement Learning with Application to Libration Point Orbits, in: AIAA Scitech 2020 Forum, no. January, 2020.

[32] K. Yanagida, N. Ozaki, R. Funase, Exploration of Long Time-of-Flight Three-Body Transfers Using Deep Reinforcement Learning, in: AIAA Scitech 2020 Forum, American Institute of Aeronautics and Astronautics (AIAA), 2020.

[33] C. J. Sullivan, N. Bosanac, Using Reinforcement Learning to Design a Low-Thrust Approach into a Periodic Orbit in a Multi-Body System (2020).

[34] A. Scorsoglio, R. Furfaro, R. Linares, M. Massari, Actor-critic reinforcement learning approach to relative motion guidance in near-rectilinear orbit, Advances in the Astronautical Sciences (2019) Paper AAS-19-441 pp. $1-20$.

[35] A. Scorsoglio, R. Furfaro, ELM-based Actor-Critic Approach to Lyapunov Vector Fields Relative Motion Guidance in Near-Rectilinear Orbit, in: 2019 AAS/AIAA Astrodynamics Specialists Conference, 2019, pp. Paper AAS-19-692 pp. 1-20.

[36] R. Furfaro, A. Scorsoglio, R. Linares, M. Massari, Adaptive Generalized ZEM-ZEV Feedback Guidance for Planetary Landing via a Deep Reinforcement Learning Approach, Acta Astronautica (In Review) (2020).

[37] H. Kwon, S. Oghim, H. Bang, Autonomous Guidance For MultiRevolution Low-Thrust Orbit Transfer via Reinforcement Learning (Preprint) AAS 21-315, in: AAS, 2021, pp. 1-16.

[38] H. Holt, R. Armellin, A. Scorsoglio, R. Furfaro, Low-Thrust Trajectory Design Using Closed-Loop Feedback-Driven Control Laws and StateDependent Parameters, in: AIAA Scitech 2020 Forum, 2020.

[39] H. Holt, R. Armellin, N. Baresi, A. Scorsoglio, R. Furfaro, Low-Thrust Trajectory Design Using State-Dependent Closed-Loop Control Laws and Reinforcement Learning, in: AAS Astrodynamics Specialist Conference, 2020, pp. 1-19.

[40] K. F. Wakker, Fundamentals of Astrodynamics, 2015. 
[41] R. H. Battin, An Introduction to the Mathematics and Methods of Astrodynamcis, AIAA Education Series, New York, 1987.

[42] J. L. Shannon, D. Ellison, C. Hartzell, Analytical Partial Derivatives of the Q-Law Guidance Algorithm, in: AAS, 2021, pp. 1-15.

[43] R. Sutton, A. Barto, Reinforcment Learning: An Introduction, MIT Press, Cambridge, 1998.

[44] I. Grondman, L. Busoniu, G. Lopes, R. Babuska, A survey of actorcritic reinforcement learning, IEEE Transactions on Systems, Man, and Cybernetics, Part C: Applications and Reviews 42 (6) (2012) 1291-1307.

[45] R. Sutton, D. McAllester, S. Singh, Y. Mansour, Policy Gradient Methods for Reinforcement Learning with Function Approximation, Advances in Neural Information Processing Systems 12 (1999) pages 10571063.

[46] D. Silver, G. Lever, N. Hess, T. Degris, D. Wierstra, M. Riedmiller, Deterministic Policy Gradient (DPG), ICML (2014).

[47] G. B. Huang, What are Extreme Learning Machines? Filling the Gap Between Frank Rosenblatt's Dream and John von Neumann's Puzzle, Cognitive Computation 7 (3) (2015) 263-278.

[48] J. Schulman, F. Wolski, P. Dhariwal, A. Radford, O. Klimov, Proximal Policy Optimization Algorithms (2017) 1-12.

[49] J. Schulman, S. Levine, P. Moritz, M. Jordan, P. Abbeel, Trust region policy optimization, 32nd International Conference on Machine Learning, ICML 20153 (2015) 1889-1897.

[50] K. J. DeMars, M. K. Jah, Probabilistic initial orbit determination using Gaussian mixture models, Journal of Guidance, Control, and Dynamics 36 (5) (2013) 1324-1335.

[51] S. Geffroy, R. Epenoy, Optimal low-thrust transfers with constraints Generalization of averaging techniques, Acta Astronautica 41 (3) (1997) 133-149. 


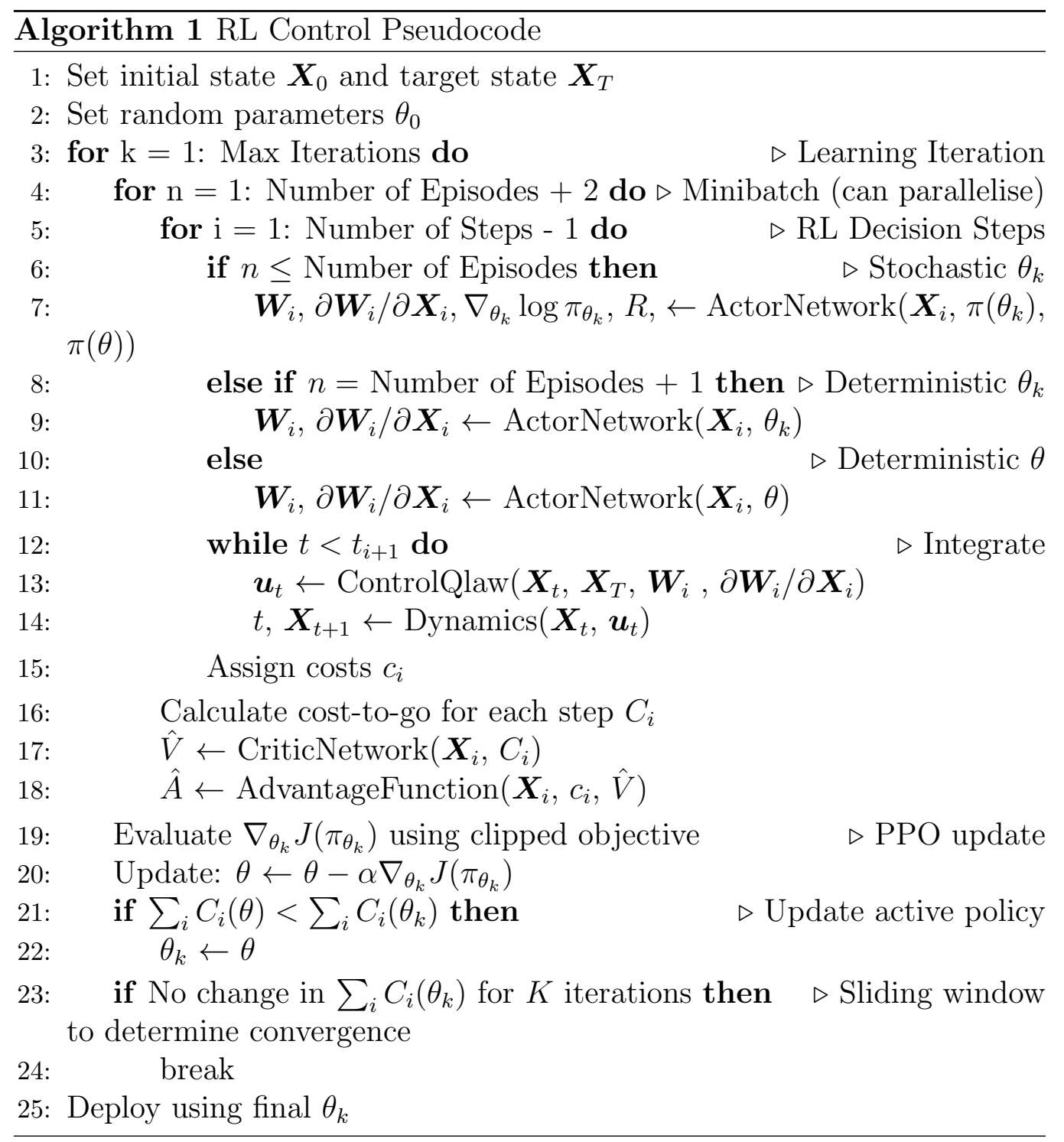



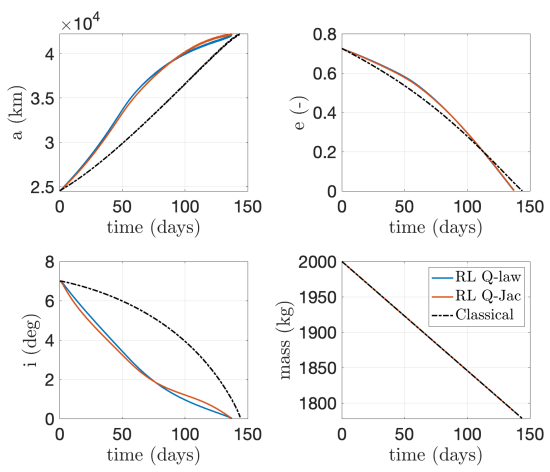

(a) Orbital Parameters

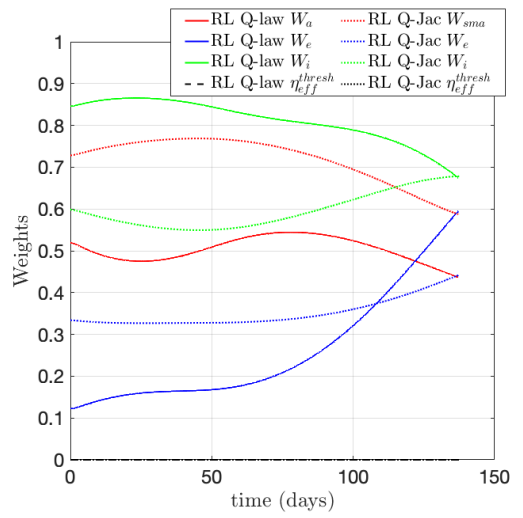

(c) Weights using $\boldsymbol{u}_{\text {original }}$ and $\boldsymbol{u}_{\text {jacobian }}$.

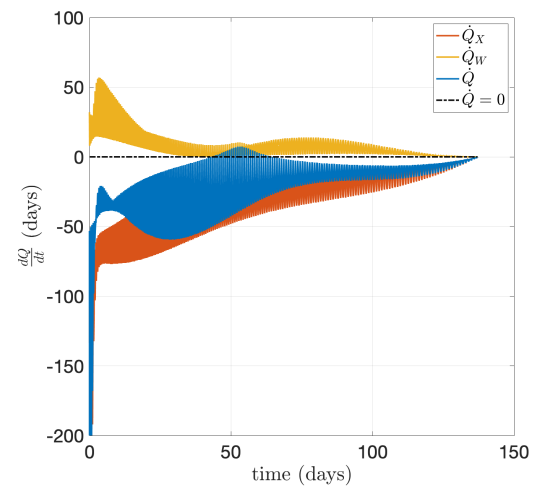

(e) $\dot{Q}$ using $\boldsymbol{u}_{\text {original }}$.

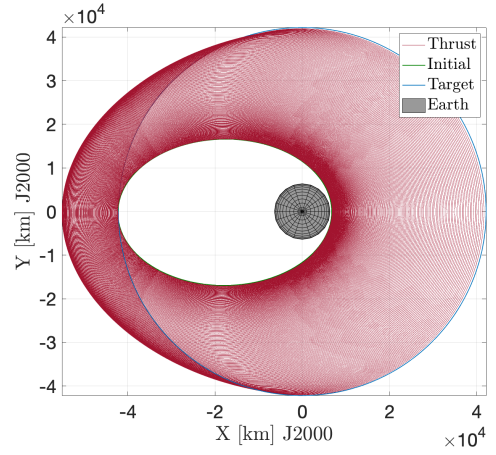

(b) Trajectory using $\boldsymbol{u}_{\text {jacobian }}$

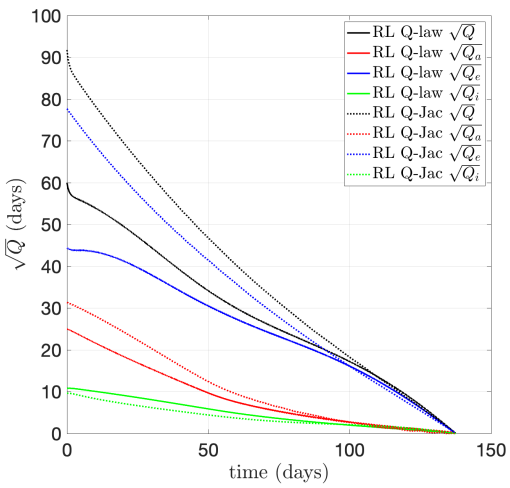

(d) Contributions to Q-law using $\boldsymbol{u}_{\text {original }}$ and $\boldsymbol{u}_{\text {jacobian }}$.

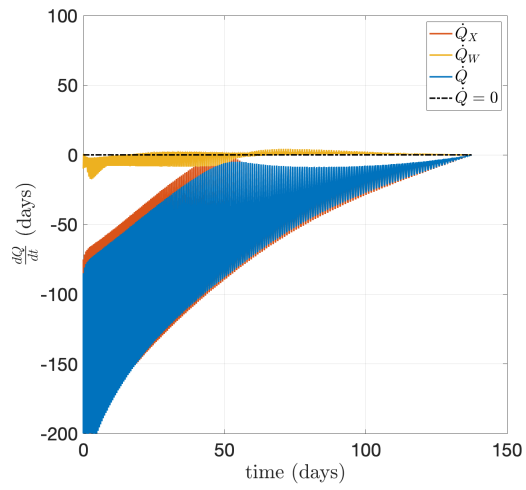

(f) $\dot{Q}$ using $\boldsymbol{u}_{\text {jacobian }}$.

Figure 4: Time-optimal GTO-GEO results breakdown for $\boldsymbol{u}_{\text {original }}$ and $\boldsymbol{u}_{\text {jacobian }}$ controls. In Fig. 4e and 4f, $\dot{Q}_{X}$ and $\dot{Q}_{W}$ refer to the contributions to $\dot{Q}$ from the state and weight variations respectively. 

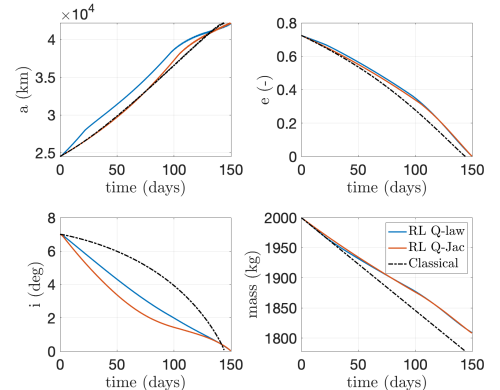

(a) Orbital Parameters

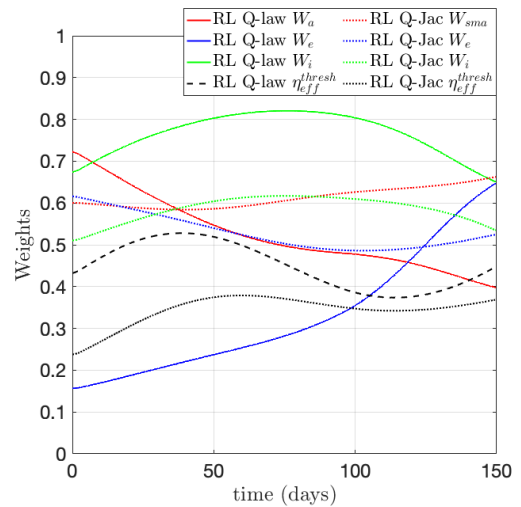

(c) Weights using $\boldsymbol{u}_{\text {original }}$ and $\boldsymbol{u}_{\text {jacobian }}$.

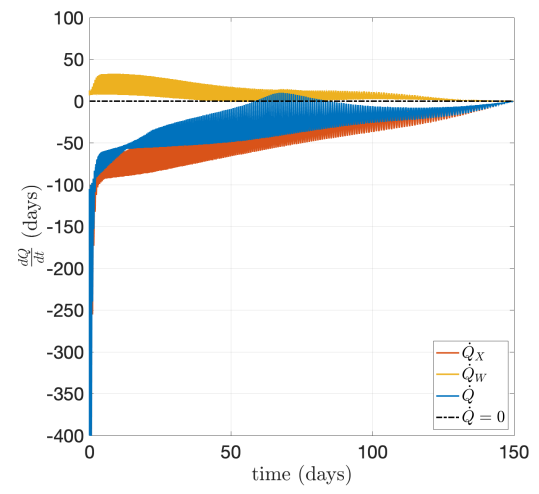

(e) $\dot{Q}$ using $\boldsymbol{u}_{\text {original }}$.

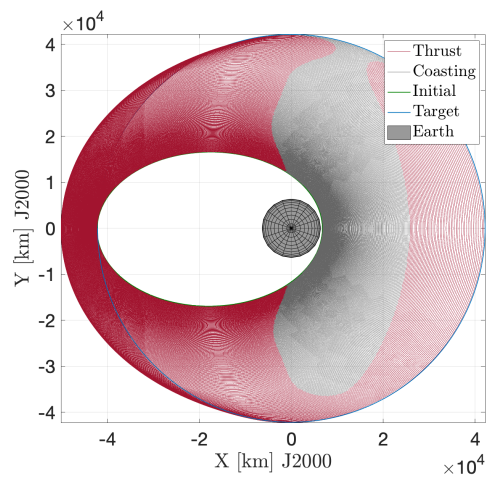

(b) Trajectory using $\boldsymbol{u}_{\text {jacobian }}$

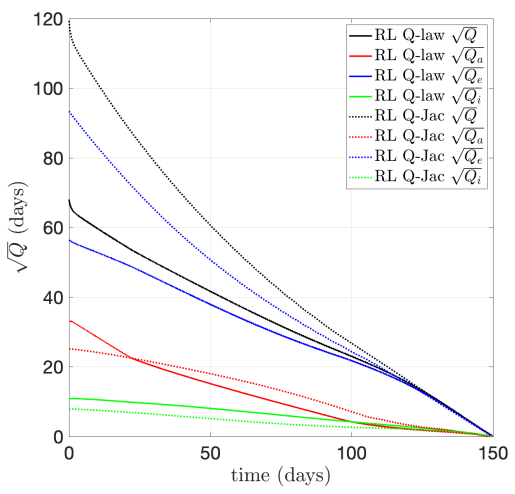

(d) Contributions to Q-law using $\boldsymbol{u}_{\text {original }}$ and $\boldsymbol{u}_{\text {jacobian }}$

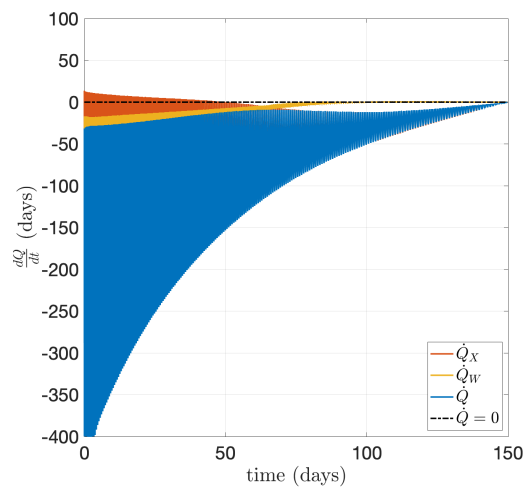

(f) $\dot{Q}$ using $\boldsymbol{u}_{\text {jacobian }}$.

Figure 5: Mass-optimal GTO-GEO results breakdown for $\boldsymbol{u}_{\text {original }}$ and $\boldsymbol{u}_{\text {jacobian }}$ controls. In 5e and 5f, $\dot{Q}_{X}$ and $\dot{Q}_{W}$ refer to the contributions to $\dot{Q}$ from the state and weight variations respectively. 


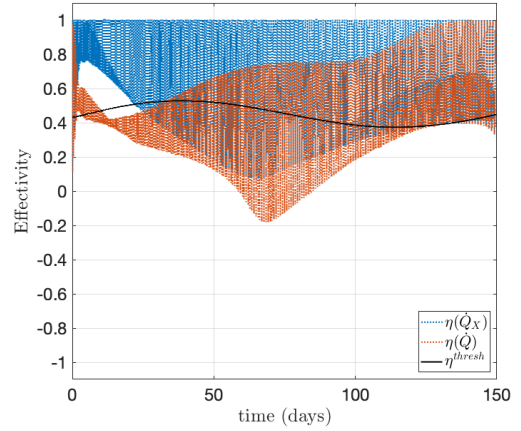

(a) $\boldsymbol{u}_{\text {original }}$

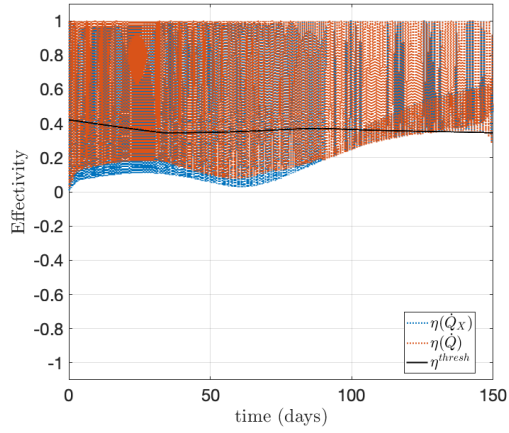

(b) $\boldsymbol{u}_{\text {jacobian }}$

Figure 6: Effectivity parameter $\eta_{a}^{\text {thresh }}$ for the mass-optimal GTO-GEO transfers. In both cases, the effectivity can be calculated using either the full knowledge of $\dot{Q}$ or using the more widely available but lesser knowledge of $\dot{Q}_{X}$ 

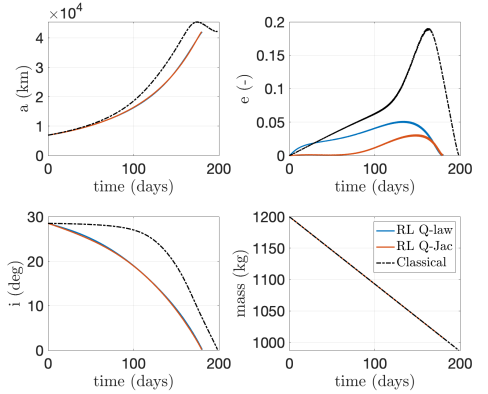

(a) Orbital Parameters

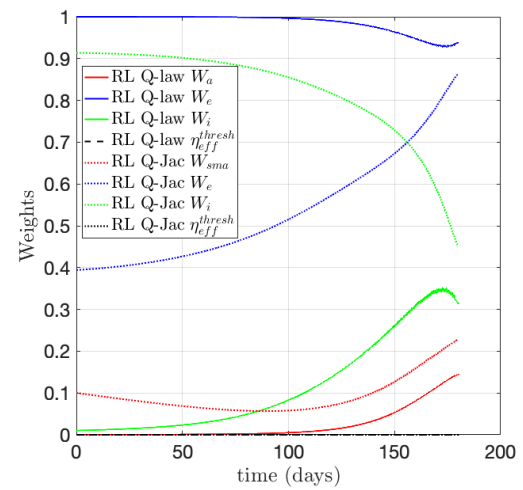

(c) Weights using $\boldsymbol{u}_{\text {original }}$ and $\boldsymbol{u}_{\text {jacobian }}$.

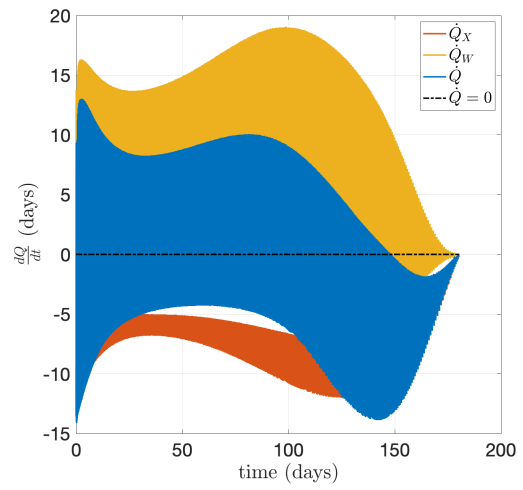

(e) $\dot{Q}$ using $\boldsymbol{u}_{\text {original }}$.

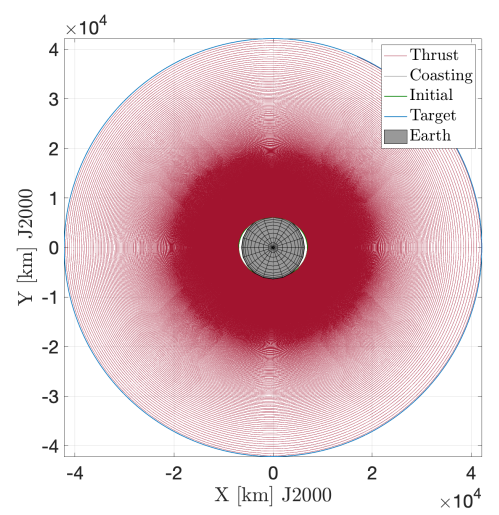

(b) Trajectory using $\boldsymbol{u}_{\text {jacobian }}$

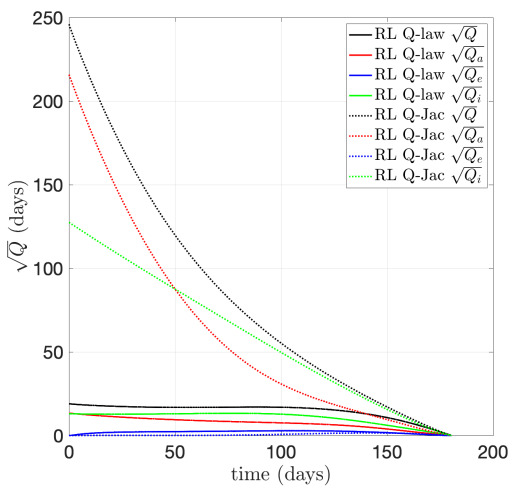

(d) Contributions to Q-law using $\boldsymbol{u}_{\text {original }}$ and $\boldsymbol{u}_{\text {jacobian }}$.

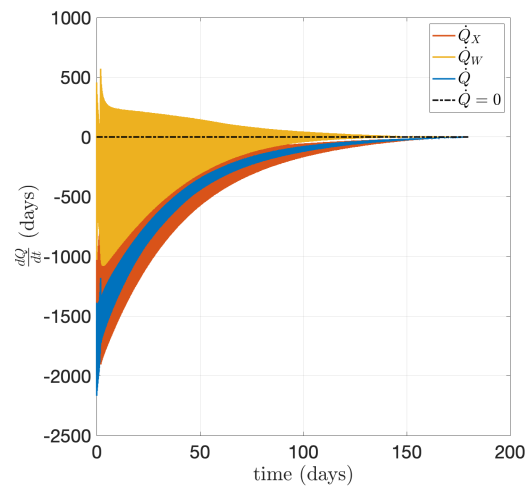

(f) $\dot{Q}$ using $\boldsymbol{u}_{\text {jacobian }}$.

Figure 7: Time-optimal LEO-GEO results breakdown for $\boldsymbol{u}_{\text {original }}$ and $\boldsymbol{u}_{\text {jacobian }}$ controls. In 7e and 7f, $\dot{Q}_{X}$ and $\dot{Q}_{W}$ refer to the contributions to $\dot{Q}$ from the state and weight variations respectively. 

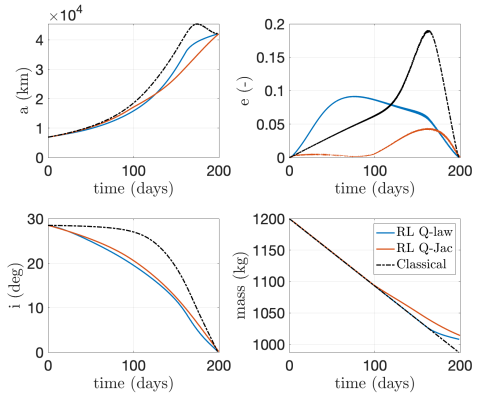

(a) Orbital Parameters

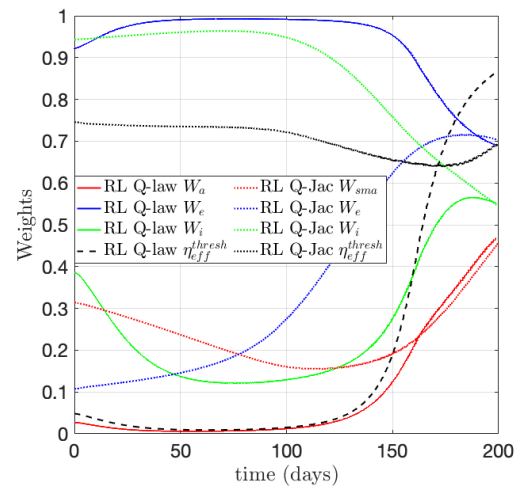

(c) Weights using $\boldsymbol{u}_{\text {original }}$ and $\boldsymbol{u}_{\text {jacobian }}$.

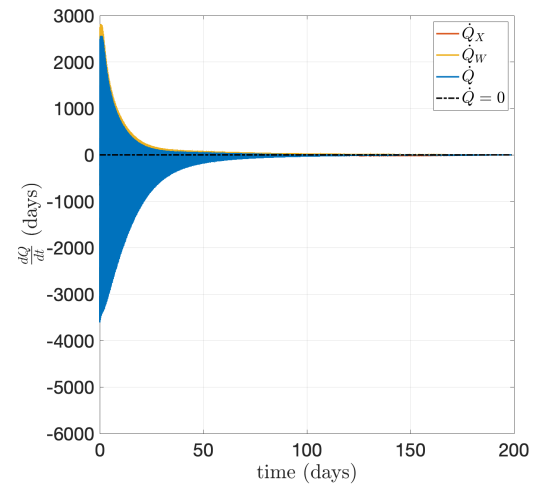

(e) $\dot{Q}$ using $\boldsymbol{u}_{\text {original }}$.

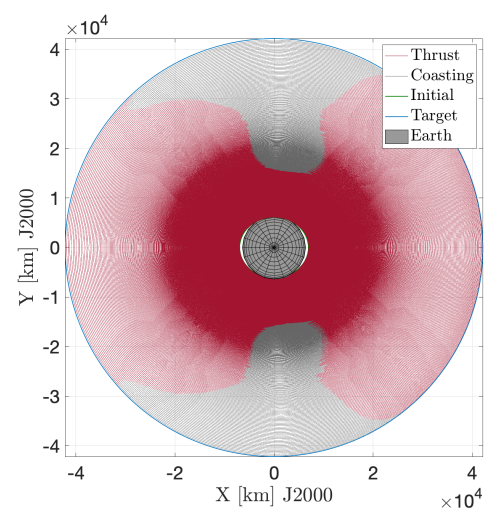

(b) Trajectory using $\boldsymbol{u}_{\text {jacobian }}$

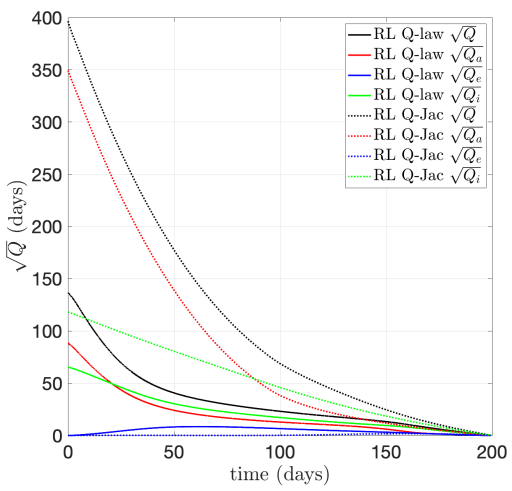

(d) Contributions to Q-law using $\boldsymbol{u}_{\text {original }}$ and $\boldsymbol{u}_{\text {jacobian }}$.

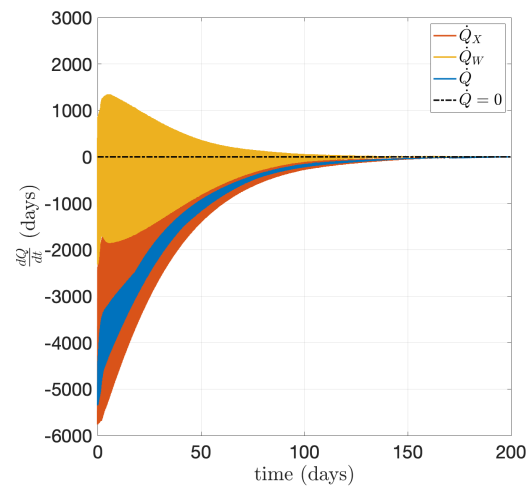

(f) $\dot{Q}$ using $\boldsymbol{u}_{\text {jacobian }}$.

Figure 8: Mass-optimal LEO-GEO results breakdown for $\boldsymbol{u}_{\text {original }}$ and $\boldsymbol{u}_{\text {jacobian }}$ controls. In 8 e and 8 f, $\dot{Q}_{X}$ and $\dot{Q}_{W}$ refer to the contributions to $\dot{Q}$ from the state and weight variations respectively. 


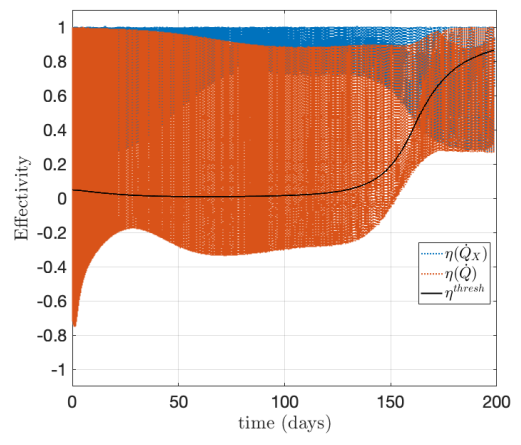

(a) $\boldsymbol{u}_{\text {original }}$

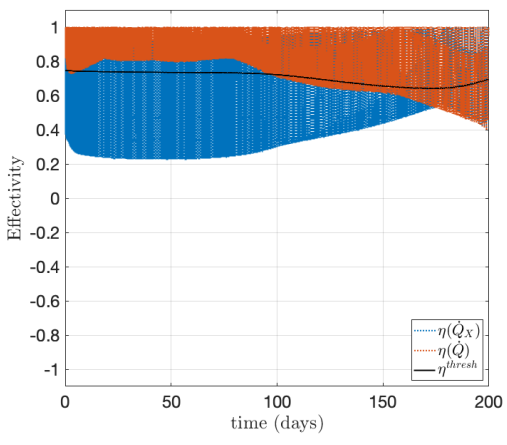

(b) $\boldsymbol{u}_{\text {jacobian }}$

Figure 9: Effectivity parameter $\eta_{a}^{\text {thresh }}$ for the mass-optimal LEO-GEO transfers. In both cases, the effectivity can be calculated using either the full knowledge of $\dot{Q}$ or using the more widely available but lesser knowledge of $\dot{Q}_{X}$

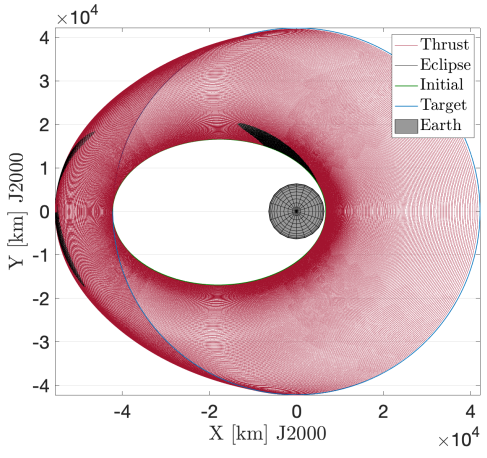

(a) GTO-GEO

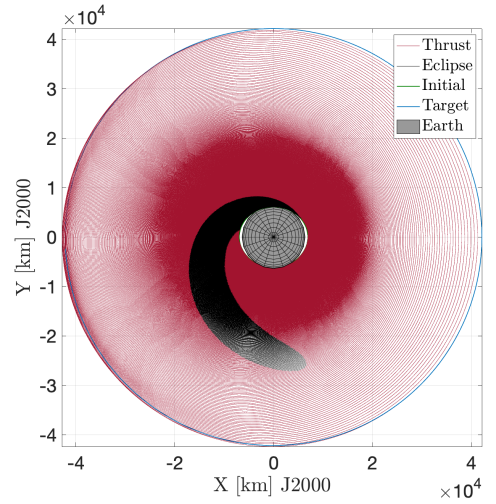

(b) LEO-GEO

Figure 10: Transfer trajectories using $\boldsymbol{u}_{\text {jacobian }}$ for GTO-GEO and LEO-GEO transfers. Black regions indicate eclipse events and red regions indicate when the engine is on. 

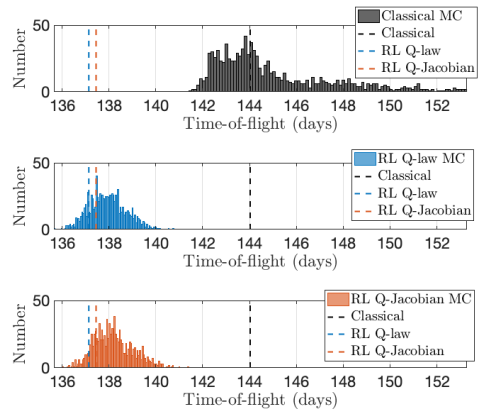

(a) GTO-GEO Time-optimal
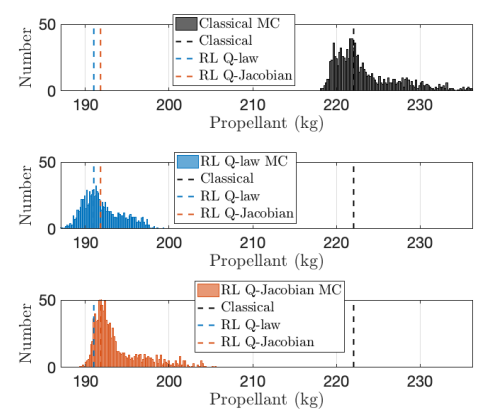

(c) GTO-GEO Mass-optimal
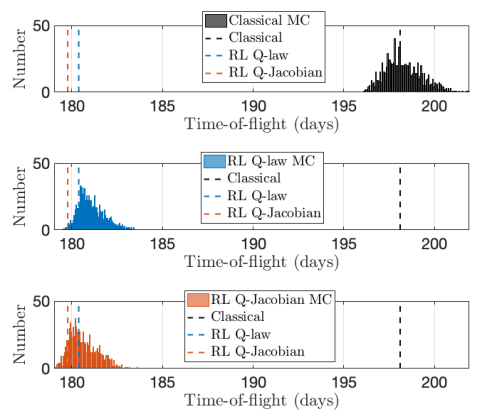

(b) LEO-GEO Time-optimal
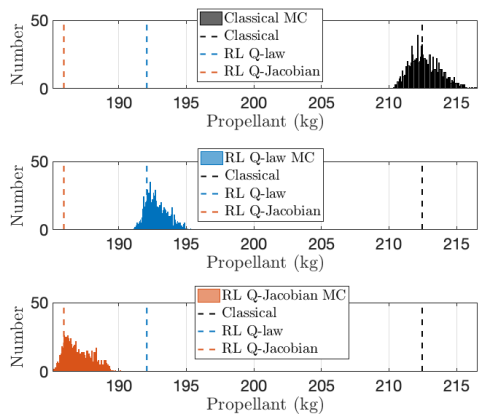

(d) LEO-GEO Mass-optimal

Figure 11: Monte Carlo Simulations with stochastic disturbances for the GTO-GEO and LEO-GEO transfers 


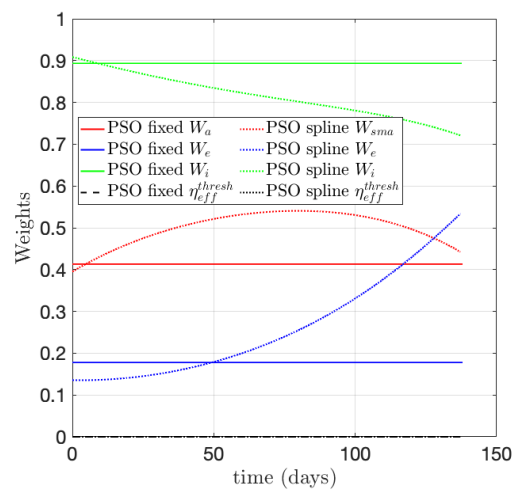

(a) Time-optimal weights.

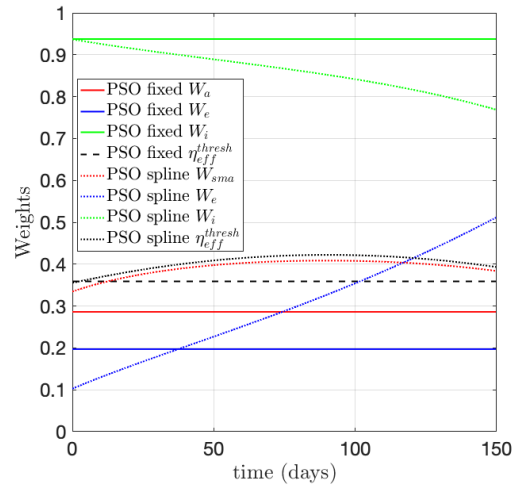

(c) Mass-optimal weights.

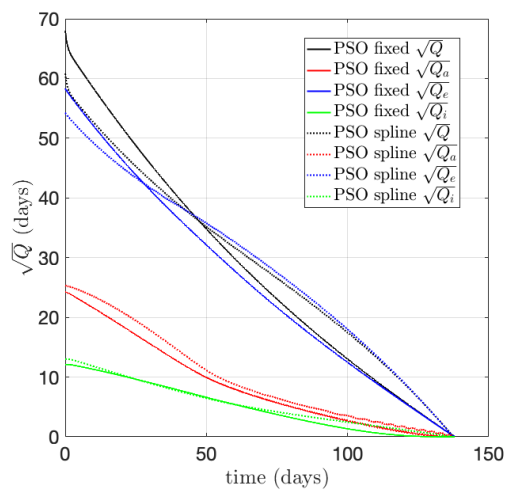

(b) Time-optimal Q-law contributions.

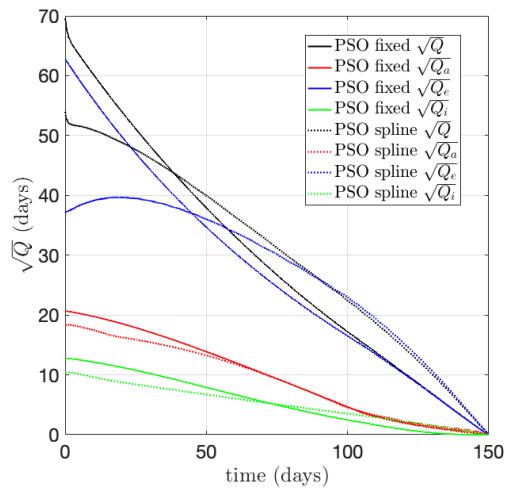

(d) Mass-optimal Q-law contributions.

Figure A.12: Particle Swarm Optimised (PSO) Q-law results for GTO-GEO transfer. Both time-optimal and mass-optimal are shown for both the PSO fixed and PSO spline approaches. In both cases the control is computed using $\boldsymbol{u}_{\text {original }}$. 


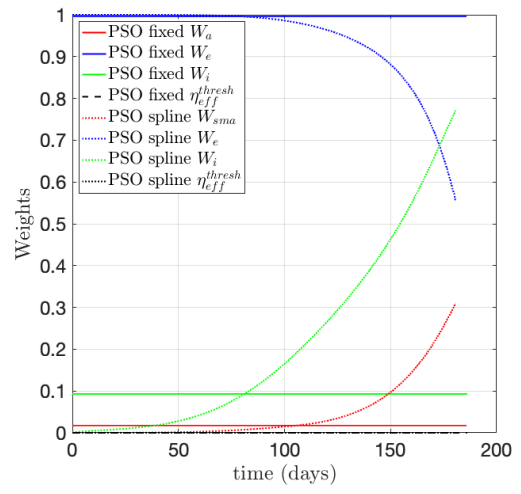

(a) Time-optimal weights.

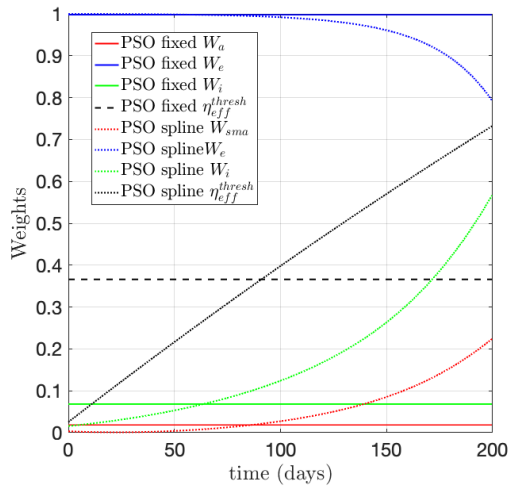

(c) Mass-optimal weights.

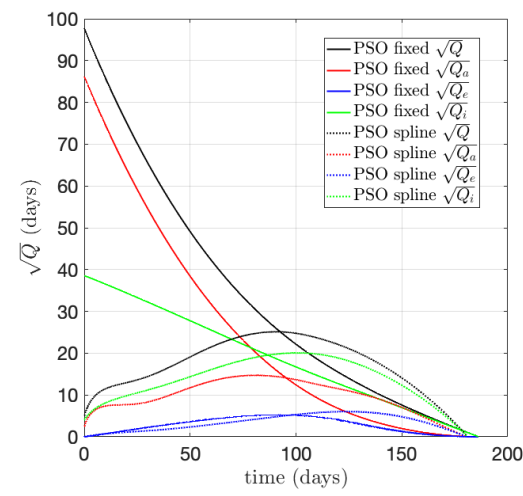

(b) Time-optimal Q-law contributions.

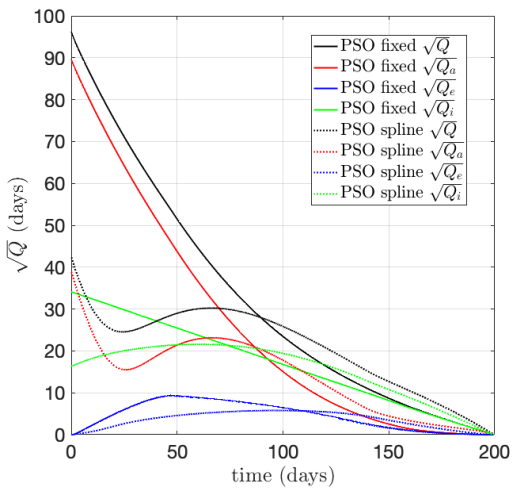

(d) Mass-optimal Q-law contributions.

Figure A.13: Particle Swarm Optimised (PSO) Q-law results for LEO-GEO transfer. Both time-optimal and mass-optimal are shown for both the PSO fixed and PSO spline approaches. In both cases the control is computed using $\boldsymbol{u}_{\text {original }}$. 